\title{
A velocity-vorticity method for highly viscous 3D flows with application to digital rock physics
}

\author{
Laurène Hume, Philippe Poncet \\ Universite de Pau et des Pays de l'Adour, E2S UPPA, CNRS, LMAP UMR CNRS-UPPA 5142, Pau, France.
}

\begin{abstract}
In this article, we present a numerical iterative method for the solution of internal viscous and incompressible flows in real porous three-dimensional bodies at their pore scale. We use the penalized formulation of the problem involving velocity and vorticity: an operator splitting allows to split apart the diffusion (inherited from Stokes equation) and the penalization phenomena (which takes into account the solid matrix). By means of the numerical analysis of the splitting, we exhibit the penalization coefficient which is actually effective. This method allows to deal only with fast-evaluation operators, that is to say scaling at most as $O(n \log n)$ where $n$ is the number of underlying grid points, such as straightforward computations of finite differences schemes or FFT solver. The numerical analysis and implementation solutions are presented, and validated on various digital rock physics geometries acquired by micro-tomography, using numerical and physical diagnostics. To enforce this validation, we also present permeability estimations of several porous samples. The simulation of transport of passive and active scalars is finally investigated in order to perform the practical upscaling to 1D models of transport and diffusion at the Darcy scale.
\end{abstract}

Keywords: Particle methods, Complex geometry, Penalization, Porous media, Digital rock physics, Geo-sciences.

\section{Introduction}

The behavior of viscous fluids in microfluidics is a matter of physics at very low Reynolds number. The Digital Rock Physics, that is to say the numerical simulations of the flow inside the poral space of rocks (or pore-scale simulations), involve complex geometries: a solid rock matrix and a fluid region, whose interface is so complex that it visually almost fills the domain (the rock sample). Managing such geometries usually leads to large computational costs, hence the need of high-performance-computing approach or conception of well-fitted numerical methods. In the present case, the model is a stationary Stokes equation combined with divergence-free condition on the velocity. On the one hand, such a flow is intrinsically useful in order to understand the properties and the mechanisms occurring inside porous media, and the Stokes problem becomes a component in coupled problems that models more complex flows, such as rheology [60], heterogeneous flows [55], reactive [50, 45, 44, 27] or multiphase flows [64]. On the other hand, a velocity field enables to compute permeability estimations [15, 22], whose accuracy is crucial for upscaling techniques. Introducing the permeability (obtained by the computation of the velocity in a representative cell) in an upscaled model is an homogenization process [32,48]. As an example, Darcy's law lies as an homogenized model for Stokes viscous flow in a porous medium. This kind of process allows to upscale complex microscopic problem without losing its major properties.

Among the different approaches to perform pore-scale simulations, one can find mainly mesh-based methods such as finite volumes [68, 16, 45], finite elements [3, 11], or grid-based methods such as finite differences or spectral methods. Other techniques can involve integral methods such as Stokeslets and boundary elements method [51, 54, $12,40]$, stochastic approach such as lattice-Boltzmann methods [65, 36], or spectral element methods [49]. In the case of internal flows inside complex geometries, mesh-based methods require a heavy pre-processing of the experimental scan in order to mesh the fluid domain, with its inherent uncertainties and errors. They also require a substantial computational time to perform matrix assembly by the appropriate variational method. One of their advantages is to strictly enforce no-slip conditions at fluid-solid interface. The grid-based methods relying on finite differences can be high-order and possibly coupled to immersed boundary methods (involving fictive domains and their inverse problems), integral methods or penalization. In order to avoid odd-even index decoupling, staggered grids [47] or mid-cell interpolations [14] are efficient techniques to reach high order and good accuracy.

In the present study, we consider the finite difference method together with a penalization technique, as introduced in [8]. Penalization can be used either to satisfy no-slip boundary conditions, up to the size of the transition layer (also called boundary layer in asymptotic analysis), or to model multi-scale porosity, leading to Brinkman models (such as Darcy-Brinkman-Stokes model) and Kozeny-Carman laws [38, 19]. In finite difference methods, the best way to satisfy a divergence-free condition is to introduce staggered grids in order to satisfy exactly $\operatorname{div} \nabla=\Delta$, which is a crucial property to compute a pressure field that leads to a divergence of velocity remaining at zero [47, 

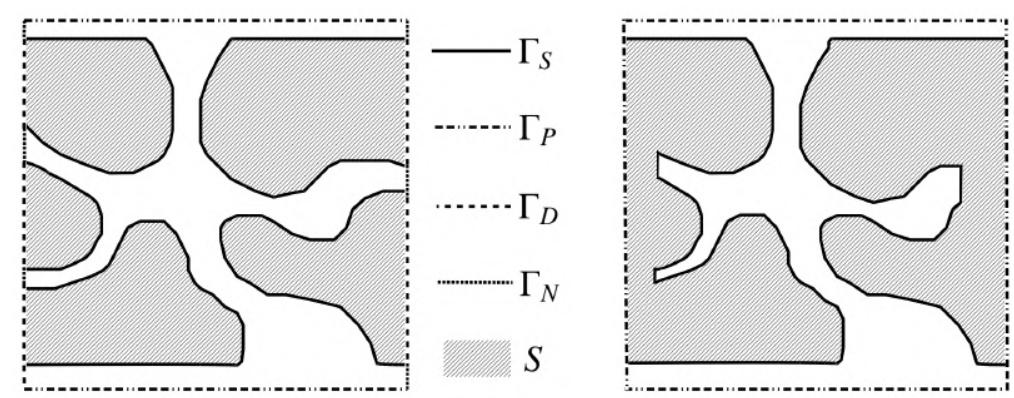

Figure 1: Example of configuration for boundary conditions on a slice of the domain (to the left) and its encapsulation in a lateral solid cell allowing full periodic boundary conditions (to the right).

73]. In practice, the geometry used to describe the solid and the fluid regions in digital rock physics is provided by X-ray tomography $[34,4]$. Since micro-tomography provides fluid/solid description on voxels (uniform grid), we have chosen to use colocated velocity so that they are aligned to experimental data on which penalization can be straightforwardly performed. Morevover, vorticity also allows to perform projections of divergence-free velocity fields, the discrete operators satisfying div curl $=0$ on colocated points, despite the extra use of memory required for a vector potential instead of using of a scalar pressure. The method developed in this article presents the solution of the stationary Stokes equation as the limit in time of the evolutionary penalized Stokes equation, with a fractional time step algorithm splitting apart diffusion, penalization and projection on divergence-free fields. Since the projection uses vorticity, there are some similarities with the penalized vortex methods described in [26, 29, 58, 31, 42] and improved in [30]. Furthermore, a projection step is not formulated as a dynamical system, and thus cannot be involved as a part of standard time-splitting method, such as Strang or Trotter splitting: the consistency of the general algorithm requires a dedicated study at a numerical analysis level.

More thoroughly, the present study introduces an iterative method for the solution of the three-dimensional incompressible Stokes equation at the pore scale of porous media :

$$
\left\{\begin{array}{l}
-\mu \Delta u=f-\nabla p \text { in } \Omega \backslash S, \\
u=\bar{u} \text { in } S, \\
\operatorname{div} u=0 \text { in } \Omega,
\end{array}\right.
$$

where $\Omega$ is the computational domain (considered as a cube in the following), $S$ denotes the solid part, $u$ is the fluid velocity, $\mu$ is its dynamic viscosity assumed to be constant, $f$ the external force and $p$ the pressure. In the solid part, we want $u$ to be equal to $\bar{u}$, which is used in practice as a lifting, for a change of variable or to consider a body in motion.

Let $\partial \Omega$ be the domain boundary and $n$ its extended outward normal field, so that $\partial \Omega=\Gamma_{P} \cup \Gamma_{D} \cup \Gamma_{N}$ is its decomposition in periodic, Neumann and Dirichlet boundary conditions, as displayed on figure 1. The solution of this problem (1) implicitly satisfies $u=\bar{u}$ on $\partial S$ and the following set of boundary conditions:

$$
\left\{\begin{array}{l}
u \text { periodic on } \Gamma_{P}, \\
u_{\perp}=0 \text { on } \Gamma_{D} \cup \Gamma_{N}, \\
u_{\|}=0 \text { on } \Gamma_{D}, \\
\frac{\partial u_{\|}}{\partial n}=0 \text { on } \Gamma_{N} .
\end{array}\right.
$$

We also consider the penalized version of the Stokes problem (1), which writes

$$
\left\{\begin{array}{l}
-\mu \Delta u^{\varepsilon}+\frac{\mathbf{1}_{S}}{\varepsilon}\left(u^{\varepsilon}-\bar{u}\right)=f-\nabla p^{\varepsilon} \text { in } \Omega, \\
\operatorname{div} u^{\varepsilon}=0 \text { in } \Omega .
\end{array}\right.
$$

This problem is defined on a simple computational box $\Omega$ whatever the complexity of the body shape.

For a constant viscosity $\mu$, splitting the operator $-\mu \Delta+\mathbf{1}_{S} \varepsilon^{-1}$ Id in two operators overcomes the numerical issues induced by its non-separability (that is to say heavy storage requirements), but this splitting often comes together with numerical instabilities due to the jump from 0 to $1 / \varepsilon$ at the fluid-solid interface. Indeed, the operator $-\mu \Delta$ is separable and its related Poisson or Helmholtz problems can be solved thanks to FFT solvers with low memory occupation and computational cost scaling as $O(N \log N)$ where $N$ is the number of grid points (such as FishPAcK [67]). We can then perform high resolution and low memory storage simulations on various geometries, including real porous media.

This article begins with the features of the penalization technique and its usual results for the penalized 3D Stokes equations in section 2, recalling existence, uniqueness and regularity of its solutions. We also recall the usual relations between vorticity, stream function and velocity, with their related boundary conditions. The section 2 ends 
with the units involed in the penalized Stokes problem and provides non-dimensional quantities and equations using characteristic dimensions.

The section 3 presents the different splitting possibilities and related issues, and the algorithm that sequentially performs penalization, diffusion and projection is finally given. The section 3.4 describes the numerical analysis of the whole algorithm: its consistency and convergence toward the solution of the Stokes problem in the fluid domain (complex geometry) with no-slip boundary conditions on the interface. In its non-dimensional formulation, the solution of the split problem is shown to converge toward the solution of the Stokes equation penalized with the coefficient $\varepsilon^{\prime}=\delta t /\left(1-e^{-\delta t / \varepsilon}\right)$. It follows that the error scales theoretically as much as size of the transition layer $O\left(\sqrt{\varepsilon^{\prime}}\right)$. This complements the existing literature, knowing that a time-splitting gives a first order error $O(\delta t)$ (cf. [35]), that the penalization of the unsplit equation induces an error $O(\sqrt{\varepsilon})$, and that our algorithm is not a straightforward time-splitting method since the projection step is not a dynamical system. This result is finally validated numerically.

This makes the present method an efficient method to study the flow inside real porous rocks or microfluidic devices, which is put in practice over real world cases in section 4. Among these examples, we consider the flow in a cylinder in order to study convergence and give meaningful diagnostics such as divergence of the velocity and residual velocities in the solid. We also consider a stack of beads that exhibits a particularly complex topology of the pore network (with a tough connectivity), and a real sandstone at high resolution. For these two study cases, physical diagnostics, such as the permeability estimation and the flow rate, are given, as well as the helicity (as a numerical diagnostic).

Finally, the section 5 uses the method developed here to the numerical simulation of coupled problems, as it fits very well to transport of passive or active species in porous media. The cases of pore-scale transport, upscaling and convergence to the solutions to the homogenized equations are investigated, using particle methods. Finally, the diffusion-transport of non-Newtonian fluids is performed, showing the robustness of the method for strongly nonlinear problems of transport.

\section{Well-known results, analysis and dimensional aspect}

\subsection{Penalization technique}

Our goal is to introduce a numerical method that avoids matrix assembly and uses as much as possible fast solvers. In order to do so, we consider a penalization of equation (1) with no-slip condition at fluid/solid interface, given by equation (3)-(2). The penalization technique is a widely used volumic method based on fictitious domain which allows to deal with obstacles in flow computation. The method has been analysed for the first time in [8], and the asymptotic analysis of the transition layer that appears in the method has been studied in $[18,17,9]$. When assembling the whole penalized matrix, the condition number is highly increased by the penalty term [41]. Some high order penalization methods have also been produced in [23], but they require a precise meshing of boundary and we can not afford this in our context of highly resolved geometries.

As shown on the penalized set of equations (3) with boundary conditions (2), the penalization formally consists in considering Stokes equation on all the domain $\Omega$ and adding a term depending on $\varepsilon<<1$ and the characteristic function $\mathbf{1}_{S}$. This volume penalization method implies that we do not have to know the exact localization of the interface between the fluid and solid parts, which is often difficult to consider as a surface.

\subsection{Well-posedness and analysis of convergence}

We recall here some results about penalization for 3D Stokes flow, by application of the results available in [21] to the present problem of penalized 3D Stokes equation, in order to focus on a well-posed problem in the following.

We first introduce $\Gamma_{S}=\partial S \backslash \partial \Omega$ that represents the fluid/solid interface that is not on boundaries of $\Omega$, and we extend the definition of the outward normal $n$ to $\Gamma_{S}$ (i.e. oriented towards the pore space). This set is useful to provide diagnostics of residual velocities on this interface. Furthermore, we introduce the $C^{2}$-bi-regularity property (cf. section 4 of [2]), which means $C^{2}$-regularity for both $S$ and its complementary in $\Omega$. This property is crucial for the analysis to be correct. In particular, the interface $\Gamma_{S}$ between solid and fluid parts must not form any non-periodic cusp at the boundary $\partial \Omega$. In the context of the present study, we also consider the lifting $\bar{u}$ that induces a velocity in the solid domain $S$, so that the final solution $u-\bar{u}$ gets rid of velocity in the solid. Moreover, the Lebesgue space $\mathbb{L}^{2}(\Omega)$ denotes the usual space of functions whose square is Lebesgue-integrable, and $\mathbb{H}^{1}(\Omega)$ is the usual Sobolev space containing generalized functions whose derivatives are in $\mathbb{L}^{2}(\Omega)$ (in case of periodic directions, one considers the Sobolev space $\mathbb{H}^{1}(\Omega)$ restricted to periodic functions in the appropriate directions). Similarly, $\mathbb{H}^{2}(\Omega)$ denotes the functions that belong to $\mathbb{H}^{1}(\Omega)$ and whose second derivatives are in $\mathbb{L}^{2}(\Omega)$.

Then the problem (3) is well-posed and converges toward the original Stokes equation (1)-(2) by means of the following theorem (whose proof is available in [21]) :

Theorem 1. Let $\varepsilon>0$. If the domain $S$ is an open set in $\Omega$ having the uniform $C^{2}$-bi-regularity property up to the boundary of $\Omega$, if the driving velocity $\bar{u} \in \mathbb{H}^{2}(S)$ in the solid $S$ is divergence-free and matches the boundary conditions 
(2), and if $f \in \mathbb{L}^{2}(\Omega)$, there exists then a unique weak solution $\left(u^{\varepsilon}, p^{\varepsilon}\right)$ to (3) such that $u^{\varepsilon} \in V(\Omega)$ and $p^{\varepsilon} \in \mathbb{L}_{0}^{2}(\Omega)$, where

$$
\begin{aligned}
& \mathbb{L}_{0}^{2}(\Omega)=\left\{w \in \mathbb{L}^{2}(\Omega), \int_{\Omega} w d x=0\right\}, \\
& V(\Omega)=\left\{u \in \mathbb{H}^{1}(\Omega), \operatorname{div} u=0 \text { on } \Omega, u=0 \text { on } \Gamma_{D}, u_{\perp}=0 \text { on } \Gamma_{N}\right\} .
\end{aligned}
$$

Moreover, there exists a unique weak solution $(u, p)$ to (1) and the weak solution $\left(u^{\varepsilon}, p^{\varepsilon}\right)$ to (3) converges weakly towards ( $u, p)$ as $\varepsilon$ goes to zero.

In the applications, we will consider samples constrained in a solid carter, eventually with a void layer on top and bottom parts. This leads to a periodic domain satisfying the hypothesis above in every case. The penalization is then an efficient way to approach the solution to Stokes flow, under the condition that the geometry is initially sufficiently well described.

\subsection{Velocity-vorticity relations}

The vorticity field $\omega$, which represents the local rotation of the fluid flow, is related to velocity field via the relation $\omega=\operatorname{curl} u$, and the vorticity is straightforwardly obtained from velocity.

There also exists different methods to get velocity from a given vorticity. For example, we have curl $\omega=$ $\operatorname{curl}(\operatorname{curl} u)=\nabla(\operatorname{div} u)-\Delta u=-\Delta u$, under the condition of incompressibility $\operatorname{div} u=0$. For a given $\omega$, one can solve $-\Delta u=\operatorname{curl} \omega$ with boundary conditions that ensure $\operatorname{div} u=0$ to get $u$. However, solving this problem can be numerically tedious because of the expected lack of regularity of $u$ at the solid/fluid interface, whose jump (respectively jump of its gradient) can lead to double (respectively single) layer singularities of $\operatorname{curl} \omega$.

An other method lies on a stream relation to avoid this issue. We define $\psi: \Omega \subset \mathbf{R}^{3} \rightarrow \mathbf{R}^{3}$ the stream function solution of $-\Delta \psi=\omega$ in $\Omega$, satisfying the following boundary conditions:

$$
\begin{cases}x=x_{\text {min }} \text { or } x=x_{\text {max }} & : \psi_{y}=\psi_{z}=\frac{\partial \psi_{x}}{\partial n}=0, \\ y=y_{\text {min }} \text { or } y=y_{\max } & : \psi_{x}=\psi_{z}=\frac{\partial \psi_{y}}{\partial n}=0, \\ z=z_{\text {min }} \text { or } z=z_{\text {max }} & : \psi_{x}=\psi_{y}=\frac{\partial \psi_{z}}{\partial n}=0,\end{cases}
$$

that ensures $\operatorname{div} \psi=0$ and $\operatorname{curl} \psi \cdot n=0$ at the boundary. These conditions can be replaced by periodic boundary conditions when needed. Consequently, we have $\Delta(\operatorname{div} \psi)=\operatorname{div}(\Delta \psi)=-\operatorname{div} \omega=0$ in $\Omega$ and $\operatorname{div} \psi=0$ on $\partial \Omega$. This equation has a unique solution equal to 0 , so $\operatorname{div} \psi=0$ everywhere. One can then set $u=\operatorname{curl} \psi$ and consequently write $\operatorname{curl} u=\operatorname{curl}(\operatorname{curl} \psi)=\nabla(\operatorname{div} \psi)-\Delta \psi=\omega$.

\subsection{Physical considerations and non-dimensional formulation}

The aim of this section is to setup the dimensionless version of the equations, that is to say to build relative (non-dimensional) variables and coordinates from absolute variables and coordinates (whose units are involved in the physical model). From a dimensional point of view, the Stokes equation (3) is an equality between $\left[\mathrm{kg} \mathrm{m}^{-2} \mathrm{~s}^{-2}\right]=$ $\left[\mathrm{Nm}^{-3}\right]$. The forces $f$ and $\nabla p$ are expressed in $\mathrm{N} \mathrm{m}^{-3}$, the dynamic viscosity $\mu$ is $\mathrm{Pas}=\mathrm{N} \mathrm{m}^{-2} s$ so that the unit of the penalization coefficient $\varepsilon$ is $m^{4} N^{-1} s^{-1}$.

We first introduce a characteristic length $L$, associated to a meaningful scale of the physical problem: a meaningful estimation of pore size in the context of Digital Rock Physics, or number of pore sizes in order to get a representative cell of material. In practice, for the simulations presented in this article, we use a width of about 10 patterns to calibrate this characteristic length $L$ (when the permeability $K$ is known, it is also possible to set $L=\sqrt{K}$ ). From the absolute space variable $X=(x, y, z)^{T}$ we introduce the dimensionless relative space variable $X^{*}=X / L$. Moreover, using the boundary conditions (4) in order to compute a velocity field with a lifting $\bar{u}$ such as $u=\operatorname{curl} \psi+\bar{u}$ leads to a flow rate $\bar{u} \cdot n$ at input/output boundaries. The characteristic velocity can thus be defined by $U=|\bar{u} \cdot n|$ and provide the dimensionless velocity $u^{*}=u / U$. It follows directly that $\bar{u}^{*}=\bar{u} / U$ is a vector basis up to its sign (typically $e_{z}$ ).

The penalized Stokes equation (3) writes in relative (non-dimensional) variables as follows:

$$
-\Delta u^{*}+\frac{\mathbf{1}_{S}}{\varepsilon^{*}}\left(u^{*}-\bar{u}^{*}\right)=f^{*}-\nabla p^{*},
$$

where $\varepsilon^{*}=\mu \varepsilon / L^{2}, f^{*}=L^{2} f / \mu U$ and $p^{*}=L p / \mu U$ are dimensionless. This stationary feature is the limit of a Reynolds number $R e=\rho U L / \mu \rightarrow 0$, the present case corresponding to an undefined volumic mass $\rho$.

Furthermore, we can add the dimensionless time $t^{*}$ so that the solution of (5) is the limit for long times of the following non-stationary Stokes equation:

$$
\frac{\partial u^{*}}{\partial t^{*}}-\Delta u^{*}+\frac{\mathbf{1}_{S}}{\varepsilon^{*}}\left(u^{*}-\bar{u}^{*}\right)=f^{*}-\nabla p^{*},
$$


whose time $t^{*}$ is dimensionless. By means of the characteristic time $T=L / U$ and the absolute time $t=t^{*} T$, the dimensional formulation of equation (6) would involve a virtual density $\rho=\mu / U L$, but not connected to any physical meaning. From now on, we consider the non-dimensional formulation and get rid of star notation in order to help readability.

\section{Penalization-Diffusion-Projection splitting of the 3D Stokes equation}

\subsection{Non-separable model and different splitting}

A direct consequence of boundary conditions (4) is that theglobal flow rate is the integral of $u \cdot n=\operatorname{curl} \psi \cdot n=0$. The field $u$ retrieved straightforwardly by $\psi$ has no flow rate, and the driving force is provided by the constant lifting velocity $\bar{u}$ that creates the desired flow rate. One can recall that its dimensionless formulation is a vector basis of norm 1 , typically $\pm e_{z}, z$ being the vertical coordinate, and satisfies trivially both $\operatorname{div} \bar{u}=0$ and $\operatorname{curl} \bar{u}=0$.

The Stokes problem (3) then rewrites $\mathcal{L} u=g_{\varepsilon}-\nabla p$ where $\mathcal{L}=-\mu \Delta+\varepsilon^{-1} \mathbf{1}_{S} I d$ with adequate boundary conditions (periodic or homogeneous Dirichlet conditions for example). This operator $\mathcal{L}$ is non-separable, as the coefficient $\varepsilon^{-1} \mathbf{1}_{S}$ is variable in space, which usually leads to numerical difficulties and expensive solver, computationally speaking. In this work, we propose to handle separately the diffusion and penalization operators in $\mathcal{L}$ with a splitting method. This kind of method is widely studied [28] to deal with coupled problems in order to get more efficient algorithms.

In order to be able to use fast solvers and operator splitting in the spirit of penalized vortex methods, we introduce an artificial dependence in time to the penalized equation (3). This gives (6) which is recalled here:

$$
\begin{cases}\frac{\partial u}{\partial t}-\Delta u+\frac{\mathbf{1}_{S}}{\varepsilon} u=g_{\varepsilon}-\nabla p & \text { in } \Omega, \\ \operatorname{div} u=0 & \text { in } \Omega,\end{cases}
$$

with adequate homogeneous boundary conditions and an initial condition, for which the lifting defined above is included in the term $g_{\varepsilon}$.

Here the pressure aims at satisfying the incompressibility condition $\operatorname{div} u=0$. It is defined up to a constant (vanishing in the evaluation of $\nabla p$ ), which can be set by requiring $p$ to have a zero mean value. Since $u$ is required to be divergence-free for every time $t$, the pressure satisfies

$$
-\Delta p=\operatorname{div}\left(\varepsilon^{-1} \mathbf{1}_{S}(u-\bar{u})\right)
$$

and is then an affine function of $u$.

In order to justify formally the consistency of the diffusion-penalization splitting, we introduce the linear operators $A$ and $B$ defined by $A u=\Delta u$ and

$$
B u=-\varepsilon^{-1} \mathbf{1}_{S} u-\nabla\left(-\Delta^{-1}\right) \operatorname{div}\left(\varepsilon^{-1} \mathbf{1}_{S} u\right),
$$

so that $\operatorname{div} B \equiv 0$ and

$$
A u+B u-B \bar{u}=\Delta u-\frac{\mathbf{1}_{S}}{\varepsilon}(u-\bar{u})-\nabla p .
$$

The problem (7) can then be written under the form

$$
\frac{\partial u}{\partial t}-(A+B) u=C \text { in } \Omega,
$$

with $C=-B \bar{u}=-g_{\varepsilon}+\nabla\left(-\Delta^{-1}\right) \operatorname{div}\left(g_{\varepsilon}\right)$ and homogeneous boundary conditions (especially for the Poisson equation defining $-\Delta^{-1}$ so that operator $B$ is indeed linear). This is the natural way to split apart diffusion and what remains of the Stokes equation.

By means of this artificial time, the idea is to numerically solve the problem with an iterative method and make the solution converge towards a stationary state. A numerical threshold then has to be set, in order to appreciate the stabilization of the iterative solution. When reached, the final solution corresponds to the stationary state of equation (7), that is to say the solution to $-(A+B) u=C$, formally equivalent to (3) for constant viscosity flows.

In the context of homogeneous linear partial differential equations, that is to say under the form $\partial_{t} u-A u-B u=0$, it is well-known that the splitting into the evaluation of two successive problems $\partial_{t} u-A u=0$ and $\partial_{t} u-B u=0$ leads to a truncation error scaling as $O\left(\delta t^{2}\right)$ over a time interval $[0, \delta t]$, which means a global error at first order. The demonstration for affine operators (or linear problems with non zero right hand side) needs adaptation and is provided in appendix A, showing that this first order consistency is still valid for our splitting of (10) between diffusion and penalization-pressure. Nevertheless, this standard approach induces some difficulties that can be overcome by the use of a three-step splitting and the use of vorticity. 


\subsection{Need of three-step splitting and vorticity formulation}

Despite the theoretical validity of the splitting described above, the numerical approximation of these operators requires to bring crucial modifications to this method. Indeed, the definition of operator $B$ in equation (9) aims at satisfying

for all $u$. Then we get

$$
\operatorname{div}(B u)=-\operatorname{div}\left(\varepsilon^{-1} \mathbf{1}_{S} u\right)-\operatorname{div} \nabla\left(-\Delta^{-1}\right) \operatorname{div}\left(\varepsilon^{-1} \mathbf{1}_{S} u\right)=0
$$

$$
\frac{\partial \operatorname{div} u}{\partial t}=\operatorname{div}(B u)-\operatorname{div}(B \bar{u})=0
$$

since $u$ is a solution of the penalization-pressure step $\partial_{t} u-B u=-B \bar{u}$. This implies that the solution remains divergence-free.

Nevertheless, an estimation of $B$ implies the numerical evaluation of the operator $-\nabla\left(-\Delta^{-1}\right)$ div . If we subscript by $h$ the numerical approximations of the these operators, the divergence of the solution is numerically computed by $-\operatorname{div}_{h} \nabla_{h}\left(-\Delta_{h}^{-1}\right) \operatorname{div}_{h}$ which is consistent to $\operatorname{div}_{h}$ if one can ensure $\operatorname{div}_{h} \nabla_{h}=\Delta_{h}$, which is not the case numerically when using standard finite difference schemes, unless using staggered grids which is not our approach since our approach is to use straightforward compatibility with experimental microCT data. Consequently, we have chosen to use the vector potential $\Psi$ solution to $-\Delta \psi=\omega$ (the stream introduced in section 2.3) where $\omega=$ curl $u$ is the vorticity. Indeed, $u=\operatorname{curl}_{h} \Psi$ satisfies algebraically both $\operatorname{div} u=\operatorname{div} \operatorname{curl} \Psi=0$ and $\operatorname{div}_{h} u=\operatorname{div}_{h} \operatorname{curl}_{h} \Psi=0 \operatorname{since}$ $\operatorname{div}_{h} \operatorname{curl}_{h} \equiv 0$, which allows to provide correct diagnostics on both the divergence and the residual velocity in the body.

It is noticeable that such a way to ensure divergence-free solutions is not formulated in terms of dynamical systems, which forbids to write it as a third step in a splitting, and requires a specific analysis to prove its consistency, which is developed below in section 3.4. One can also notice that it would be equivalent to recover the velocity by means of the Poisson equation $-\Delta u=\operatorname{curl} \omega$, but the strong lack of regularity of curl $\omega$ for direct numerical simulation of real pore-scale flows forbids to use this technique. Moreover, it would be quite similar to diffuse the velocity and then compute the vorticity to perform the projection on divergence-free velocity fields, or compute the vorticity and diffuse it before building the divergence-free velocity. We have chosen the second technique, but the first one would be valid as well.

Finally, this leads to introduce the following three steps over a time step $\left[t_{n}, t_{n+1}\right]$ with $t_{n}=n \delta t$ with an initial condition $u_{n}$. The first step, dealing with the penalization, reads

$$
\frac{\partial u}{\partial t}+\frac{\mathbf{1}_{S}}{\varepsilon}(u-\bar{u})=0 \text { in } \Omega,
$$

which can be solved exactly:

$$
\widetilde{u}^{n+1}=e^{-\mathbf{1}_{S} \delta t / \varepsilon}\left(u^{n}-\bar{u}\right)+\bar{u}
$$

but breaks the zero divergence. The diffusion step comes in second:

$$
\frac{\partial u}{\partial t}-\Delta u=0 \text { or any }-\nabla \phi \text { in } \Omega
$$

By applying the curl operator to this equation, we get

$$
\frac{\partial \omega}{\partial t}-\Delta \omega=0 \text { in } \Omega .
$$

with $\omega=\operatorname{curl} u$ which is divergence-free. Explicit or implicit method can be chosen to solve this diffusion problem. In our study, we use an implicit Euler scheme, which does not involve any stability condition and can be easily implemented by means of a FFT solver. Each time step then consists in solving

$$
-\delta t \Delta \widetilde{\omega}^{n+1}+\widetilde{\omega}^{n+1}=\widetilde{\omega}^{n} \text { in } \Omega
$$

with $\widetilde{\omega}^{n}=\operatorname{curl} u^{n}$.

The velocity field can finally be recovered by means of a stream function satisfying the equation $-\Delta \psi=\widetilde{\omega}^{n+1}$ with boundary conditions (4). One gets the final velocity by the straightforward curl computation $u^{n+1}=\operatorname{curl} \psi$, which gives a divergence free field. The section 3.4 shows that this projection defines a scheme consistent with a penalized Stokes equation, satisfying both a residual velocity under control (converging toward zero as $\delta t$ tends to zero) and a strict divergence-free feature. 

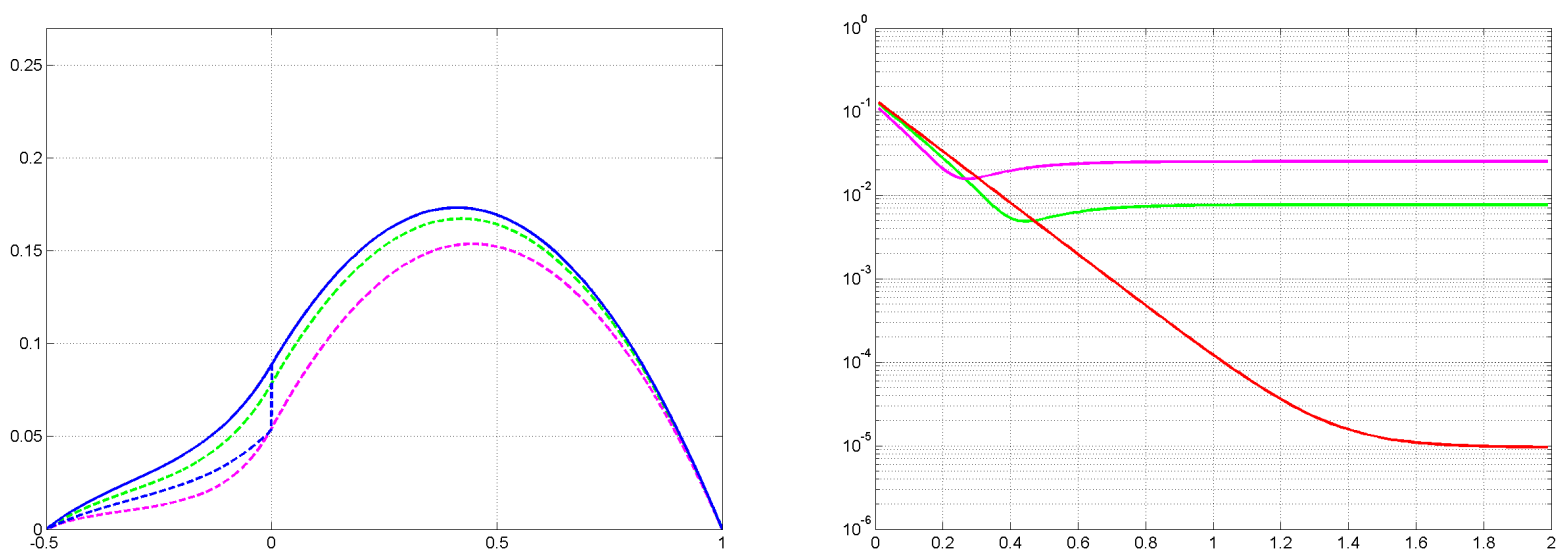

Figure 2: Convergence toward the problem penalized by $\varepsilon^{\prime}=\delta t /\left(1-e^{-\delta t / \varepsilon}\right)$, involving a discretization of 1000 points over the interval $[-1 / 2,1]$ over 200 time steps. The time step is $\delta t=10^{-2}$ and $\varepsilon=\delta t / 2$ in order to visually distinguish the curves. The interval [ $\left.-1 / 2,0\right]$ is penalized. The curve resulting from our computations is displayed in blue on the left picture (solid line is obtained after diffusion step, dashed line is obtained after penalization step). The reference curves are the exact solution when penalized by $\varepsilon$ (in pink), by $\delta t$ (in green) and by $\varepsilon^{\prime}$ is red. The right picture shows, with the same color legend, the maximum errors, that is to say the difference between our computation and the reference solutions.

\subsection{Resulting algorithm}

The successive numerical resolution of the previous partial differential equations can be summarized by the following algorithm [33] :

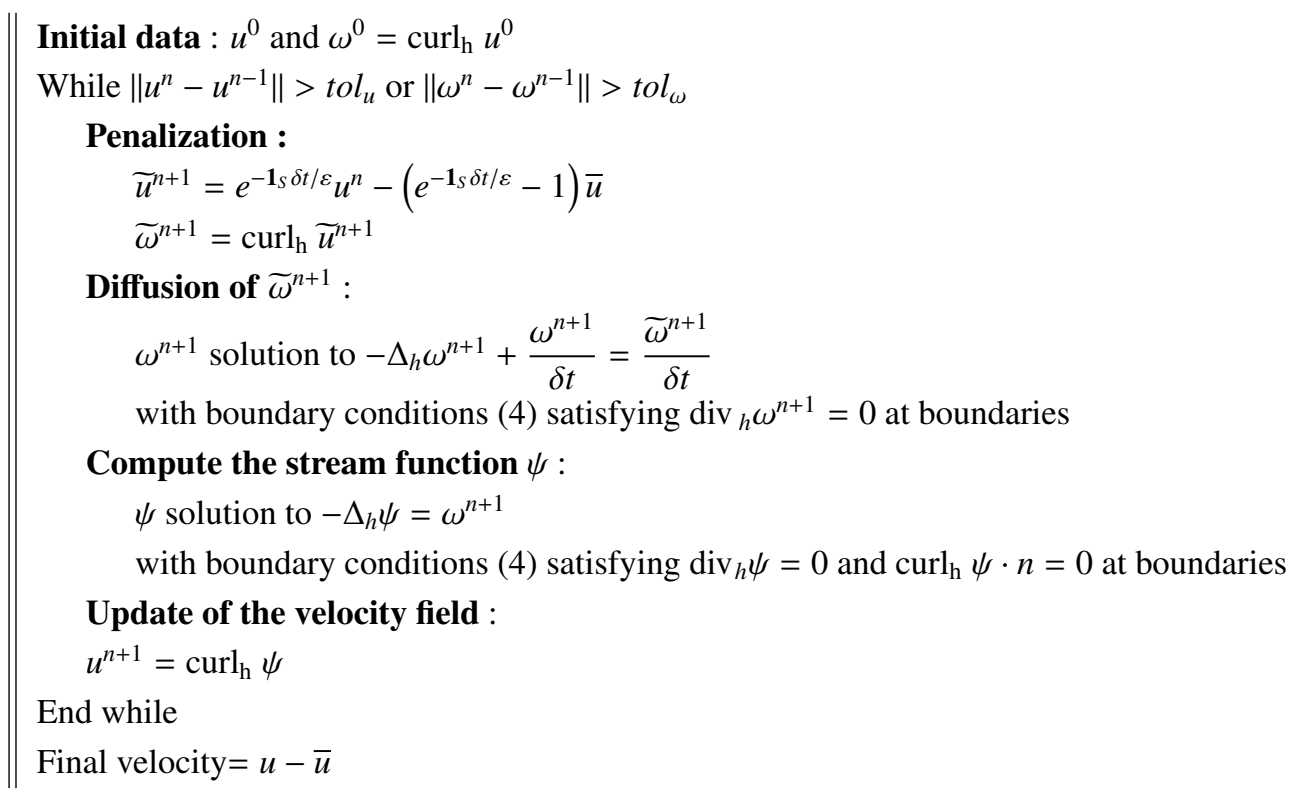

Discrete versions of differential operators are here denoted with the index ${ }_{h}$. In this algorithm, direct resolution is used for the non-stationary penalization, which means there is no linear system to solve for this operator. The stationary diffusion operator $-\Delta+\delta t^{-1} \mathrm{Id}$ is in fact separable, to the opposite of the initial problem operator $\mathcal{L}=$ $-\Delta+\varepsilon^{-1} \mathbf{1}_{S}$ Id arising in the non-splitted problem.

For the implicit vorticity diffusion, as well as for the stream function, we then use Fishpack FFT (Fast Fourier Transform) solver [67]. This solver is efficient in terms of computation time and memory storage, as it does not require to assemble any matrix. As a comparison, if $N$ denotes the number of grid points in one direction, the complete algorithm (16) requires $7.25 N^{3}+O\left(N^{2}\right)$ stored real values (since the solid characteristic function can be stored as short integers). If the initial (i.e. un-split) problem is solved with MuDPACK, which holds among the most efficient elliptic solvers (Algebraic Multi-Grid method) [1], an equivalent algorithm would require $38 N^{3}+O\left(N^{2}\right)$ values. If $N=1000$, our algorithm then uses around 58GB, while an Algebraic Multi-Grid algorithm such as MudPack would use up to 304GB.

\subsection{Numerical analysis of the splitting}

The algorithm (16) can be formally written as a sequence $u^{n}$ defined by the following recurrence:

$$
u^{n+1}=\Pi\left(\mathcal{D}\left(\operatorname{curl}\left(e^{-\mathbf{1}_{S} \delta t / \varepsilon}\left(u^{n}-\bar{u}\right)+\bar{u}\right)\right)\right),
$$


where

$$
\Pi(\zeta)=\operatorname{curl}\left(\left(-\Delta^{-1}\right) \zeta\right)
$$

for which the operator $(-\Delta)^{-1}$ takes the right hand side of the Poisson equation with the boundary conditions inherited from the stream function (4) and gives its solution. In other words, this is the linear operator $f \rightarrow u$ where $u$ is the solution of $-\Delta u=f$. In the same spirit, one defines $\mathcal{D}=(I d-\delta t \Delta)^{-1}$ denoting the diffusion process obtained by the Implicit Euler scheme, still with the same boundary conditions. The linear application $\Pi$ allows to get a divergencefree velocity field from a vorticity, and the operator $\mathcal{D}$ aims at diffusing the vorticity.

If this sequence converges, its limit is the fixed point $u$ satisfying

$$
u=\Pi\left(\mathcal{D}\left(\operatorname{curl}\left(e^{-\mathbf{1}_{s} \delta t / \varepsilon}(u-\bar{u})+\bar{u}\right)\right)\right) .
$$

Consequently, one gets

$$
\begin{aligned}
(u-\bar{u})-\delta t \Delta(u-\bar{u}) & =-\bar{u}+(I d-\delta t \Delta) u \quad(\text { since } \Delta \bar{u}=0) \\
& =-\bar{u}+\mathcal{D}^{-1}(u) \\
& =-\bar{u}+\mathcal{D}^{-1}\left(\Pi\left(\mathcal{D}\left(\operatorname{curl}\left(e^{-\mathbf{1}_{s} \delta t / \varepsilon}(u-\bar{u})+\bar{u}\right)\right)\right)\right) \\
& =-\bar{u}+\mathcal{D}^{-1}\left(\operatorname{curl}\left(\left(-\Delta^{-1}\right)\left(\mathcal{D}\left(\operatorname{curl}\left(e^{-\mathbf{1}_{s} \delta t / \varepsilon}(u-\bar{u})+\bar{u}\right)\right)\right)\right)\right) \\
& =-\bar{u}+\operatorname{curl}^{2}\left(\left(-\Delta^{-1}\right)\left(e^{-\mathbf{1}_{s} \delta t / \varepsilon}(u-\bar{u})+\bar{u}\right)\right) \\
& =-\bar{u}-\Delta\left(\left(-\Delta^{-1}\right)\left(e^{-\mathbf{1}_{s} \delta t / \varepsilon}(u-\bar{u})+\bar{u}\right)\right)+\nabla \operatorname{div}\left(\left(-\Delta^{-1}\right)\left(e^{-\mathbf{1}_{\delta} \delta t / \varepsilon}(u-\bar{u})+\bar{u}\right)\right) \\
& =e^{-\mathbf{1}_{\delta} \delta t / \varepsilon}(u-\bar{u})-\nabla(p \delta t)
\end{aligned}
$$

where by definition we set

$$
p=-(\delta t)^{-1} \operatorname{div}\left(\left(-\Delta^{-1}\right)\left(e^{-\mathbf{1}_{S} \delta t / \varepsilon}(u-\bar{u})+\bar{u}\right)\right) .
$$

In the fluid domain where $\mathbf{1}_{S}=0$, after division by $\delta t$, the fix point $u$ satisfies consequently the Stokes equation

$$
-\Delta u+\nabla p=0
$$

with the correct input/output boundary conditions $u \cdot n=0$ so that its lifting sataisfies $(u+\bar{u}) \cdot n=\bar{u} \cdot n$ or periodic.

In the solid domain where $\mathbf{1}_{S}=1$, the equation (20) reads $-\delta t \Delta(u-\bar{u})+\left(1-e^{-\delta t / \varepsilon}\right)(u-\bar{u})=-\nabla(p \delta t)$ so that once divided by $\delta t$, one gets

where

$$
-\Delta u+\frac{1}{\varepsilon^{\prime}}(u-\bar{u})+\nabla p=0
$$

$$
\varepsilon^{\prime}=\delta t /\left(1-e^{-\delta t / \varepsilon}\right)
$$

By putting together the equations obtained respectively on fluid and solid domains, the limit of the sequence (17) is solution to a penalized Stokes equation (3) penalized by $\varepsilon^{\prime}$. While it is quite obvious that penalization/diffusion splitting is consistent, this result proves that the penalization/diffusion/projection is also consistent to the stationary penalized Stokes equation, despite the projection that is not a dynamical system.

In order to get a good accuracy by penalization around the interface, the coefficient $\varepsilon^{\prime}$ has to be chosen as small as possible. This leads to choose low values for $\delta t$. We can notice that the case of $\delta t<<\varepsilon$ is useless in practice since we have $1-e^{-\delta t / \varepsilon} \simeq \delta t / \varepsilon$ at order 1: this would involve an extremely small time step and consequently a prohibitive simulation time.

But in the general case, the penalized Stokes equation can be written under the form

$$
-\Delta u+\frac{1}{\varepsilon^{\prime}}(u-\bar{u})+\nabla p=0 .
$$

with $\varepsilon^{\prime}=\delta t /\left(1-e^{-\delta t / \varepsilon}\right)$.

The convergence toward the solution to the problem penalized by $\varepsilon^{\prime}$ is displayed on figure 2. This $1 \mathrm{D}$ test case is an equation defined over the interval $[-1 / 2,1]$, with a penalization region $[-1 / 2,0]$, so we get a Poiseuille flow on $[0,1]$ with a slip at $x=0$. The parameters are set to $h=1.5 \times 10^{-3}\left(10^{3}\right.$ cells $), \delta t=10^{-2}$ and $\varepsilon=5 \times 10^{-3}$. The errors and the exact solutions obtained for penalization coefficients $\varepsilon, \delta t$ and $\varepsilon^{\prime}$ are plotted on this figure. This clearly shows the consistency with a penalization by $\varepsilon^{\prime}$.

Furthermore, in the case of $\varepsilon \ll \delta t$, one gets $e^{-\delta t / \varepsilon} \simeq 0$ and consequently the developed scheme is then consistent with a penalization coefficient of the type $1 / \delta t$. The choice made for $\delta t$ value is then crucial because it does also represent the penalization coefficient. Numerically, we actually observe that the ratio $\delta t / \varepsilon$ only has a low influence on convergence properties inside solid part, whereas $\delta t$ value needs to be low to obtain a good convergence. Moreover, the penalization coefficient is then $1 / \delta t$, which leads to a transition layer $\sqrt{\delta t}$ : this is coherent with the velocity jump regularization (after penalization) due to diffusion on one time step. In the following, the ratio between penalization parameter and time step will always be set to $\varepsilon=\delta t / 2$. 


\begin{tabular}{|c|c|}
\hline Grid resolution & Computational time per iteration \\
\hline$\left(2^{5}\right)^{3}$ cells & $0.024 \mathrm{sec}$ \\
\hline$\left(2^{6}\right)^{3}$ cells & $0.170 \mathrm{sec}$ \\
\hline$\left(2^{7}\right)^{3}$ cells & $1.4 \mathrm{sec}$ \\
\hline$\left(2^{8}\right)^{3}$ cells & $15 \mathrm{sec}$ \\
\hline
\end{tabular}

Table 1: Computational time for each iteration in cylinder simulation depending on the resolution.

a)

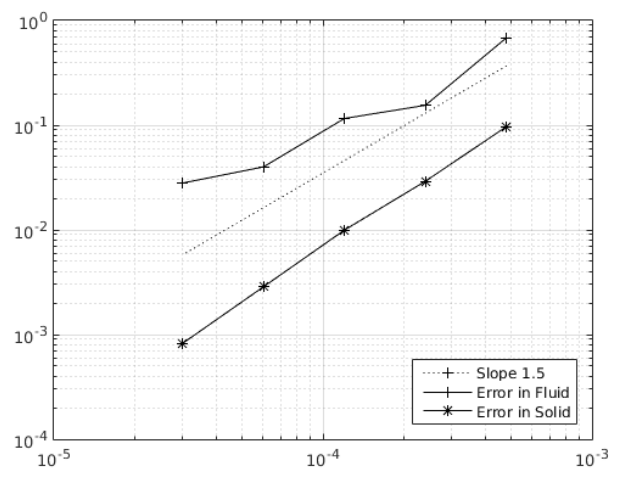

c)

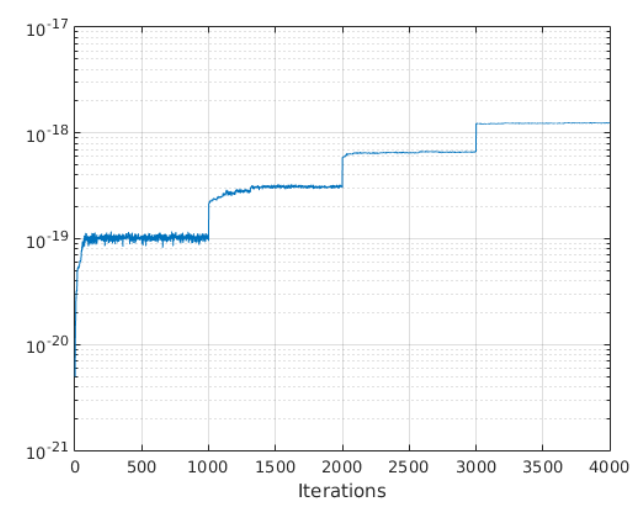

b)

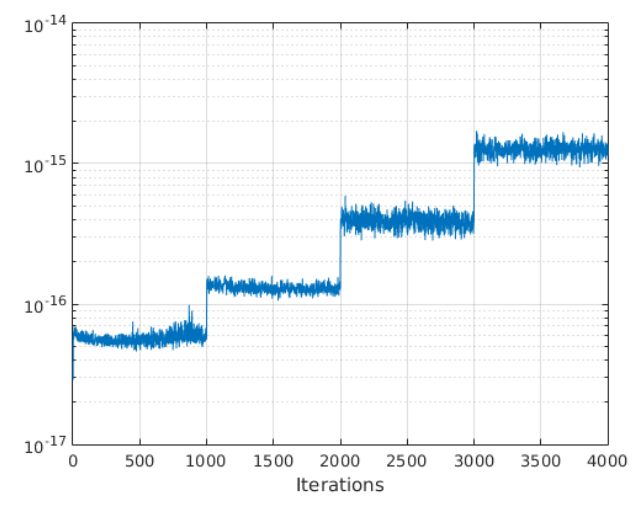

d)

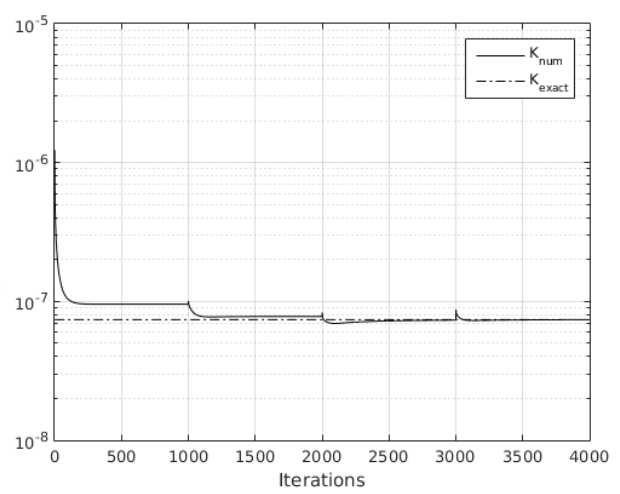

Figure 3: Convergence diagnostics for the cylinder geometry: Residual velocities in the solid and in the fluid with respect to the grid size (a) for $2^{5}, 2^{6}, 2^{7}$ and $2^{8}$ cells in each direction; Relative residual velocity at the interface (b) with respect to the number of iterations; Norm of divergence (c) and permeability (d) along with its theoretical value.

\section{Applications to microfluidics and Digital Rock Physics}

This method can be directly applied to fluid flow inside real porous domains. In our study, we present results of fluid simulation inside geometries coming from X-ray tomography. In our case, the differentiation between pore and solid spaces is crucial, such that the final image needs to contain only binary information (for each voxel, solid or not). From the geometry, we compute the flow inside the pores. We also propose a numerical estimation of permeability, which is a commonly used parameter of interest for porous media $[63,50,32]$. For all the simulations described in this section, we consider the non-dimensional formulation of the Stokes equation.

\subsection{Computation of a flow inside a circular cylinder}

The Stokes flow in a cylinder of radius $R$ for a viscous fluid is a well-known example. The permeability in its dimensional formulation is defined following Darcy law proportionality by $K=\phi \mu\langle u>/\langle f-\nabla p>$ (the brackets denote spatial average in the fluid domain and $\phi$ is the domain porosity). This can be written equivalently $K^{*}=\phi<u^{*}>/<f^{*}-\nabla p^{*}>$ in relative (non-dimensional) formulation, recovering the dimensional permability by $K=K^{*} L^{2}$

For an prescribed down-sided vertical force, we can exhibit its exact solution

$$
u(x, y, z)=-\frac{\left(R^{2}-x^{2}-y^{2}\right) f}{4} e_{z}
$$




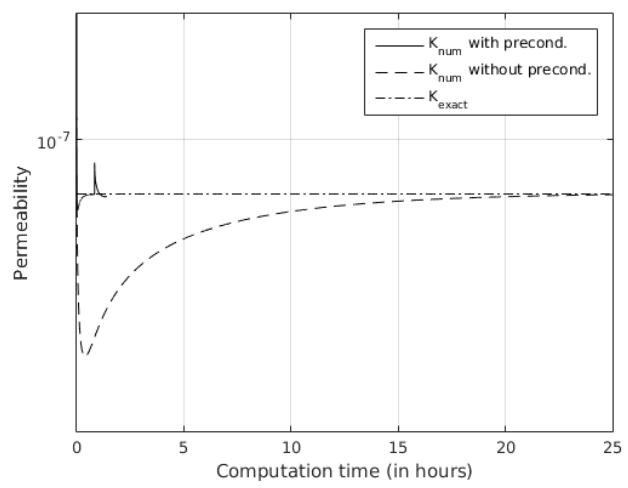

Figure 4: Permeability estimation on cylinder validation test case with respect to computation time. With preconditioning, an accurate estimation is given in few minutes, while several hours are needed without preconditioning to get a similar range.

b)
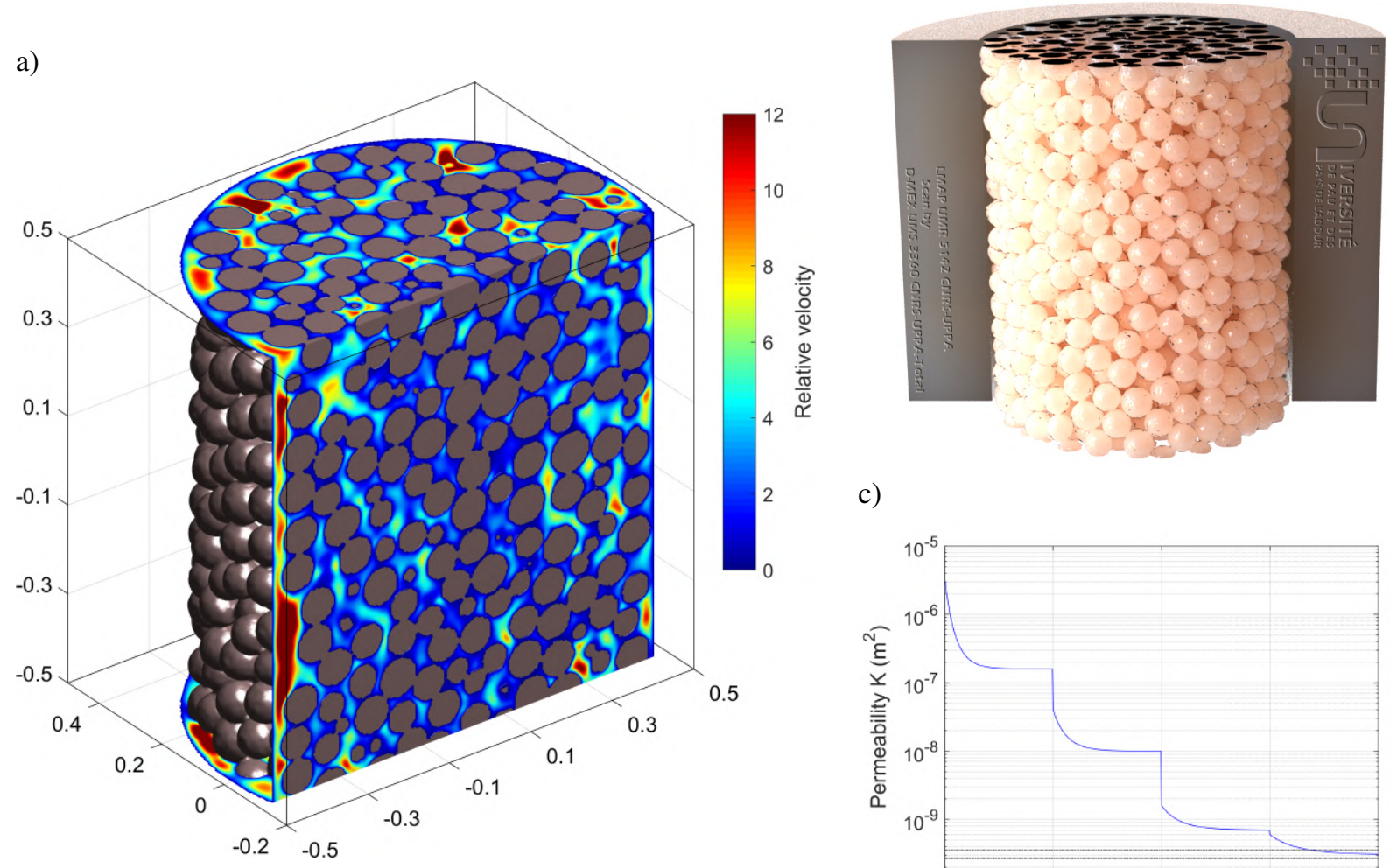

c)

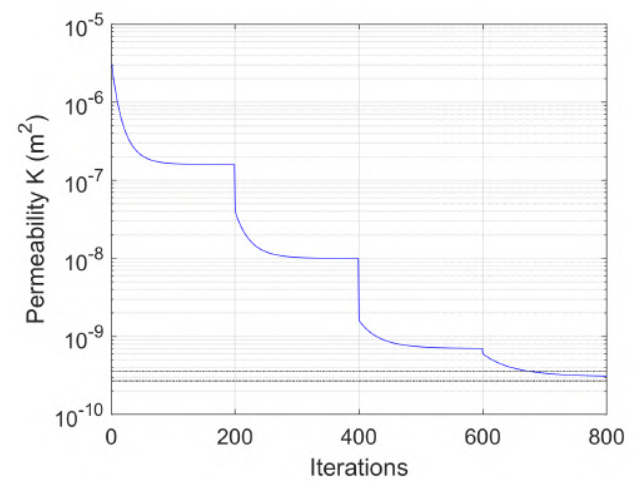

Figure 5: Beads stack geometry: Relative velocity norm (a), the geometry with its cylindrical cell (b), and resulting evolution of permeability with respect to the number of iterations, using three steps of preconditioning with grid size of $2^{5}, 2^{6}, 2^{7}$ and finally $2^{8}$ cells in each direction (c).

where $e_{z}$ is the unit basis vector in the $z$ direction (the axis of the cylinder). For the cylinder, the permeability value is then equal to $K=R^{2} / 8$.

Numerically, the averaging of $-\Delta u$ can be used in place of the force for a flow with fixed flow rate, that is to say when the driving force is not explicitly set such as for penalized problems.

The figure 3 shows relative errors and permeability estimation for each iteration of the algorithm, for a cylinder whose radius represents $10 \%$ of the cubic domain length. We have performed 1000 iterations on 4 successive grids, from the coarser $\left(2^{5}\right.$ cells in each direction) to the finer $\left(2^{8}\right.$ cells in each direction). This preconditioning process consists in computation with the algorithm on coarse grid, then interpolating the resulting fields on a twice finer grid by means of an interpolating kernel. These interpolated fields are then used as initial fields for computation on finer grid. This preconditioning method leads to step-shaped errors and estimations, as the result after 1000 iterations for a grid is used as an initial value for the following finer grid. As displayed in figure 3.a), the order of convergence is $3 / 2$.

The residual velocities in the solid body and on the fluid/solid interface are respectively defined by $\|u\|_{\mathbb{L}^{2}(S)} /\|u\|_{\mathbb{L}^{\infty}(\Omega)}$ 
and $\|u\|_{\mathbb{L}^{2}\left(\Gamma_{S}\right)} /\|u\|_{\mathbb{L}^{\infty}(\Omega)}$ where $\Gamma_{S}=\partial S \backslash \partial \Omega$ represents the interface (considered as a surface) between pore space and solid matrix. Since most of the residual error is produced by the transition layer, the residual velocity at the interface is a meaningful quantification of convergence error, here displayed on figure 3.b).

Moreover, the figure 3.c) shows $\|\operatorname{div}(u-\bar{u})\|_{\mathbb{L}^{2}(\Omega)}$ and the figure 3.d) displays values of the permeability $K$. The errors show a good convergence, residual velocity at interface and divergence are close to machine precision. The converged estimation of permeability is also quite accurate : the converged estimation exhibits $1.3 \%$ of error compared to the theoretical value.

It is important to notice that the iterations for the coarser grids correspond to very small computational time (see table 1). We then observe a factor around 8 for each refinement, and the results for the 3 preconditioning grids are obtained in less than 6 minutes. Furthermore, the computational cost of one iteration does not depend on the complexity of the geometry, but some cases may require a larger number of iterations. Figure 4 also depicts the efficiency of preconditioning, as it leads to a good approximation of permeability value in much less computation time than without preconditioning.

\subsection{Internal flow around beads stack}

In this section, the presented geometry corresponds to a real synthetic sample composed of stacked smooth microspheres. The nominal diameter of each bead is $553 \pm 11 \mu \mathrm{m}$, such that we have around 10 spheres included in the diameter of the cylindrical cell. These beads are made of dry soda lime glass and their configuration results in a completely connected porous network, exhibiting a complex topology. These beads are constrained by a cylindrical impermeable carter, displayed in grey on figure 5.a.

The 10 bead width sample is just enough to be representative of the generality of bead stacking, so the characteristic length is set to $L=7.7 \mathrm{~mm}$, which is also the width of our computational domain. The X-Ray tomography use a physical voxel size of around $30 \mu \mathrm{m}$, providing a set of 256 images at the resolution $256 \times 256$ over an area of size $L \times L$. It follows that our simulations can fit exactly the experimental setup by using $256^{3}$ cells of a domain rescaled by $L$. The virtual time step is equal to $\delta t=h^{2} / 120$ such that $\delta t / \varepsilon=2$ and the Fourier number is $1 / 120$. The resulting relative velocity is displayed on figure 5.b, and exhibits some privileged paths through the bead stack.

The numerical simulation of flow through the beads is made on a geometry resolution with 257 grid points per direction (256 cells). A down-sided vertical force is imposed as a gravitational force, such that the fluid flows through the network with a driving velocity $\bar{u}=-e_{z}$ in relative coordinates. In order to have coherent geometry and boundary conditions, the domain is made periodic in all directions by virtually cutting a thin layer at top and bottom. By this method, the top and bottom are completely considered as fluid part and the domain is set periodic in the vertical direction (thanks to the solid carter, it is already periodic on the lateral directions). Concerning the numerical convergence, as in section 4.1, the preconditioning method is also used here, for grid resolutions from $2^{5}$ to $2^{8}$ voxels in each direction. The norm of the resulting relative velocity field is displayed on figure 5.b. The maximum residual velocity at interface is about $10^{-3}$, while the maximum divergence is bounded by $10^{-18}$ (the numerical zero, that is to say we check that flow remains divergence-free algebraically). Moreover, in order to complete these diagnostics, we introduce the non-dimensional helicity [43] defined by

$$
H(t)=\frac{L}{U^{2}} \int_{\Omega}(u(t)-\bar{u}) \cdot \omega(t) \mathrm{dv}=\int_{\Omega}\left(u^{*}(t)-\bar{u}^{*}\right) \cdot \omega^{*}(t) \mathrm{dv},
$$

which should be conserved over time. The non-dimensional flow rate $Q_{z}$ through horizontal slices $S_{z}$ is defined by

$$
Q_{z}=\frac{1}{U} \int_{S_{z}} u(x, y, z)-\bar{u} \mathrm{dx} \mathrm{dy}=\int_{S_{z}} u^{*}(x, y, z)-\bar{u}^{*} \mathrm{dx} \mathrm{dy}
$$

and its mean value $\bar{Q}$. For the present simulation, the maximum errors on helicity $H$ and flow rate $\left|Q_{z}-\bar{Q}\right| /|\bar{Q}|$ are respectively bounded by $10^{-10}$ and $10^{-6}$.

At a physical level, experiments have been made by the D-MEX CNRS-UPPA-Total Unit UMS3360 and show that the studied sample exhibits a permeability around $310^{-10} \mathrm{~m}^{2}$ [34]. In order to investigate variability of the results due to the experimental process, a permeability measurement has been made, leading to a value of $3.610^{-10} \mathrm{~m}^{2}$, then the sample has been shaken and another measurement has been performed, leading to a second value of $2.710^{-10} \mathrm{~m}^{2}$. The numerical permeability is is between these values, as shown on figure 5.c.

\subsection{High resolution simulation inside a Castlegate sandstone}

The last sample used as a validation for our numerical method is a real Castlegate sandstone sample. This highly porous rock (with a porosity around $21 \%$ ) is found in particular geological formations in the USA. It is commonly used for physical and geological studies, in the context of enhanced oil recovery for example [39]. In the literature, the order of magnitude for the permeability of this kind of rock is around $10^{-12} \mathrm{~m}^{2}$. 


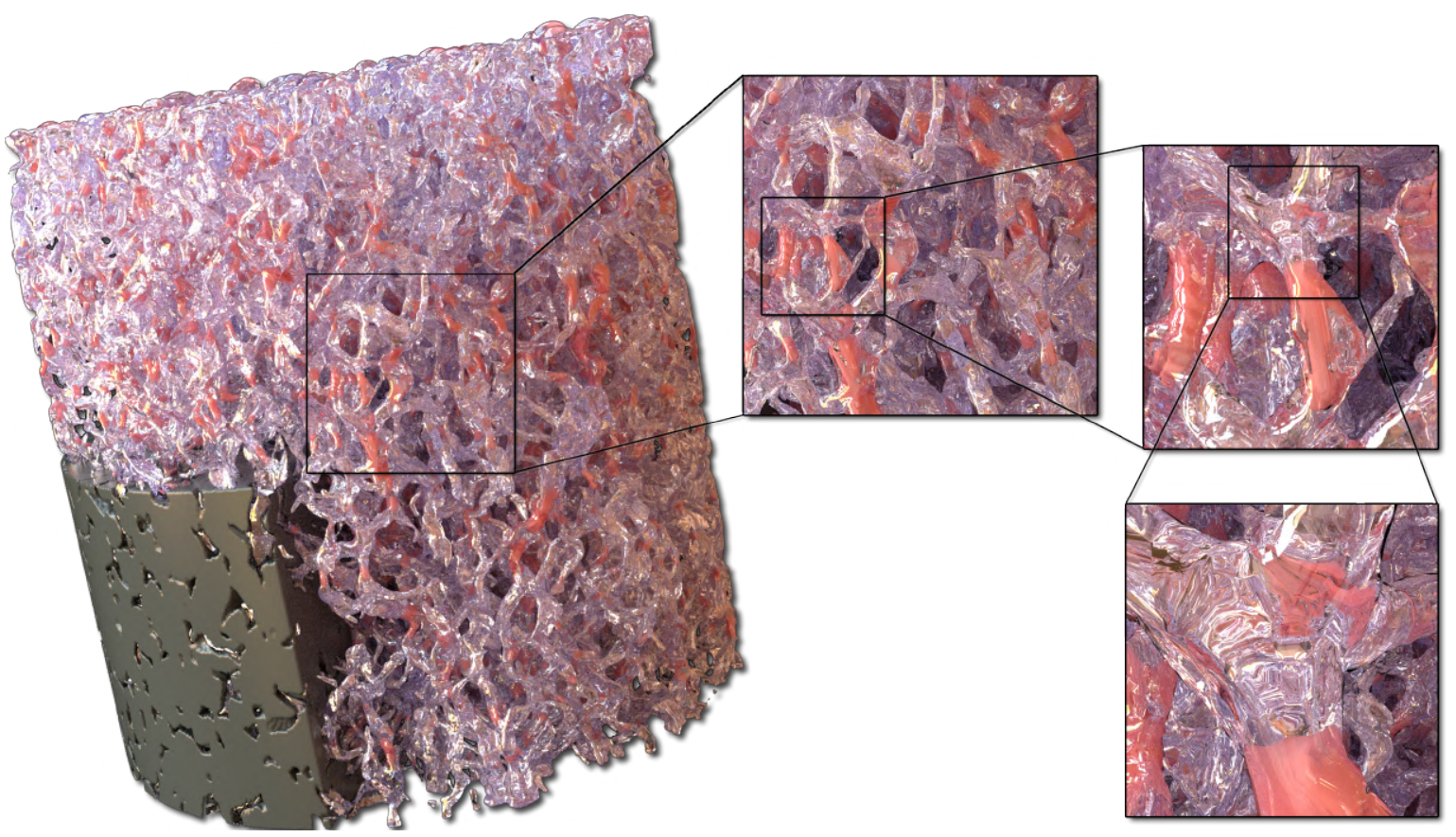

Figure 6: Castlegate sandstone: body at the top left corner, pore geometry at the top right corner, and percolating pores $(x$ such as $u(x)>0)$ showing the pore throat in red ( $x$ such as $\left.|u(x)|>u_{\max } / 4\right)$ on the bottom picture. The pictures only focus on the sample part: it is included in a solid carter that completes the cubic domain $\Omega\left(\Omega=[-0.5,0.5]^{3}\right.$ as displayed in top figures).

b)
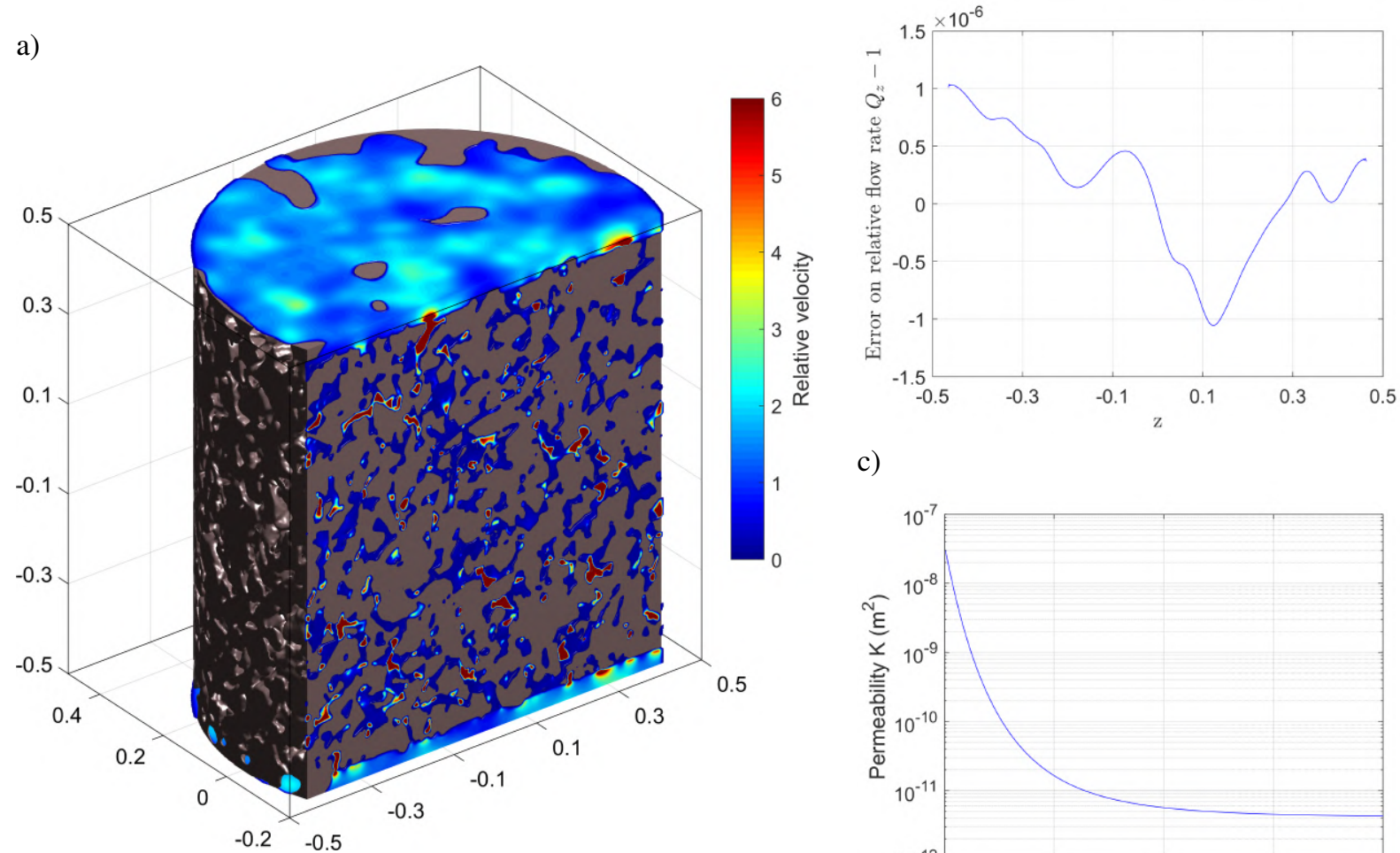

c)

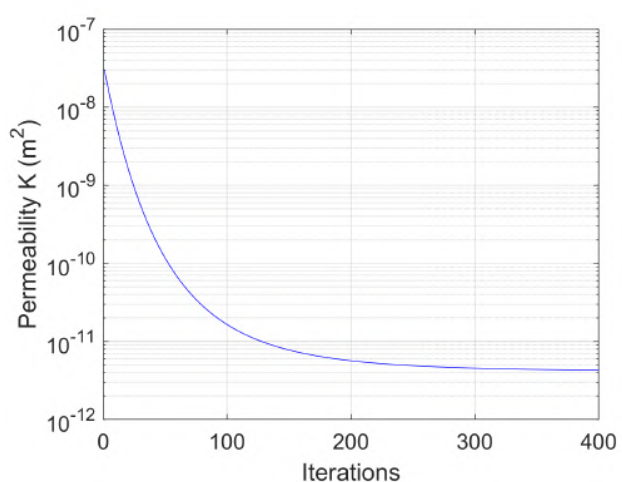

Castlegate sandstone geometry: Relative velocity norm (a), error on flow rate by slices with respect to the $z$ relative coordinate (b), and resulting evolution of permeability with respect to the number of iterations (c).

The considered geometry is a $1.8 \mathrm{~mm}$ large cylindrical sample constrained in an impermeable carter, shown in top pictures of figure 6 . The physical voxel size is approximatively $3.5 \mu \mathrm{m}$. Moreover, a grain pattern occurs around 120 times in a circular section, with a mean surface of $S_{g r}=0.0225 \mathrm{~mm}^{2}$. The characteristic length for material representativeness is set to 12 grain typical width, that is to say $L=12 \sqrt{S_{g r}}=1.8 \mathrm{~mm}$ which fits to the sample physical size. 
The present case involves a finer resolution than the previous samples: the grid contains 513 points per direction, in order to get more accurate information about the porous network, and $\delta t=h^{2} / 60$. In this case, the simulation requires more memory storage than for the cylinder and beads stack cases. Furthermore, for such rock geometry exhibiting a large collection of small pores, involving subgrids for preconditioning would be very inaccurate since it would fill artificially many pores and ruin the velocity field coarse estimation.

Consequently, we have not performed any preconditioning, and we have obtained after 400 iterations a permeability of $410^{-12} \mathrm{~m}^{2}$, whose evolution is displayed on graph 4.2.c (even though not completely converged). The final relative velocity field is shown on figure 4.2.a with surface plots, on figure 6 with a volume plot, showing the maximal values of the velocity in the pore throats, along with several levels of zooms showing the necessity to perform high resolution simulations. The resulting error of flow rate per slice displayed on graph 4.2.b scales around $410^{-6}$ in relative coordinates. Moreover, as for the previous simulations, the flow is still algebraically divergence-free.

\section{Simulations involving coupled physics}

This section describes the numerical simulations of transport phenomenon using the velocity field computed by the method there above, in three different cases. The considered model is the transport of a vector $C(x, t) \in \mathbf{R}^{d}$, following the set of equations

$$
\left\{\begin{array}{l}
\frac{\partial C}{\partial t}+\operatorname{div}(u \otimes C)-\alpha \Delta C=0 \\
-\Delta u+\nabla p+\frac{\mathbf{1}_{S}}{\varepsilon}(u-\bar{u})=0 \\
\operatorname{div} u=0
\end{array}\right.
$$

in $\Omega \times[0, T]$, where

$$
\operatorname{div}(u \otimes C)=\sum_{i=1}^{d} \sum_{j=1}^{3} \frac{\partial\left(u_{j} c_{i}\right)}{\partial x_{j}} e_{i}=(u \cdot \nabla) C,
$$

since $\operatorname{div} u=0$ (here the components of $C$ are denoted $c_{i}$ with lowercase notation), $e_{i}$ being the $i$-th vector of the basis.

The first case deals with pure transport of a passive scalar, using a non-remeshed particle method. This allows to check whether particle of fluids initially remain in the poral space or not. This would not be achievable if particles were remeshed, and is a meaningful validation since as soon as the normal velocity at the fluid/solid interface is no longer zero, the particles escape the poral space.

The second case also a diffusion-transport, but using a remeshed particle method, with a particle description of $C$. This allows accurate pure-transport and diffusion-transport simulations, and check whether the averaged pore-scale simulation is compatible to its upscaled one-dimensional model or not.

The two first cases involve a constant viscosity and pure transport, that is to say without diffusion $(\alpha \equiv 0)$, and the second case involves a constant diffusion. The third and final case introduces full coupling between the equations of the set (32), that is to say deals with the transport of an active scalar. It deals with the modeling of rheology, here the transport and of a shear-thinning fluid, whose viscosity depends on $C$ and on $u$ at the same time. This is consequently necessary to recompute the velocity field anytime that $C$ is modified. Due to the physical nature of this last simulation, dimensional quantities and their related units are re-introduced.

\subsection{Transport of passive scalars using non-remeshed particle methods}

In this section we are interested in the transport of three passive scalars, denoted as the three components of a vector $C(x, t) \in \mathbf{R}^{3}$ and following the pure transport model (32). This example is setup in order to both bring another validation of the flow computation and build a velocity field in order to perform simulation of coupled physics in the following sections. Indeed, performing a non-remeshed particle simulation allows to check whether the particles of fluid are able to follow a path in the poral space or not, avoiding being trapped in the boundary layer (slowing dramatically particles) or worse in the solid. This challenging test shall not accumulate the geometrical discrepancy due to the remeshing and the numerical errors in the velocity computation in order to validate this last one.

The computational box is the unit box $[-1 / 2,1 / 2]^{3}$. The initial condition for each component of $C$ is one inside a cylinder of height 5/128 (20 cells among the 512 vertical cells of the simulation) and radius 0.18 , respectively centered at $(x=0.3, y=0),(x=-0.2, y=0.3)$ and $(x=-0.2, y=-0.3)$, and zero outside of these cylinders.

In the present work, we consider the particle formulation of the transport problem of equation (32). Particle methods have been widely studied and used in the last decades to compute transport equations in fluid mechanics, mainly because their Lagrangian form makes the convective terms vanish, as well as the corresponding CFL stability condition $[25,13,7]$, so that large time steps can be performed. The Navier-Stokes equations under their vorticity formulation exhibit a transport of vorticity, whose particle formalism has given birth to the vortex method, based on transport of pointwise vortices. These methods are quasi-linearly scaling, even in their parallel [61] or sub-grid [26] implementations, robust and accurate [24,37], which is particularly interesting for large 3D computations [52, 20]. 
a)

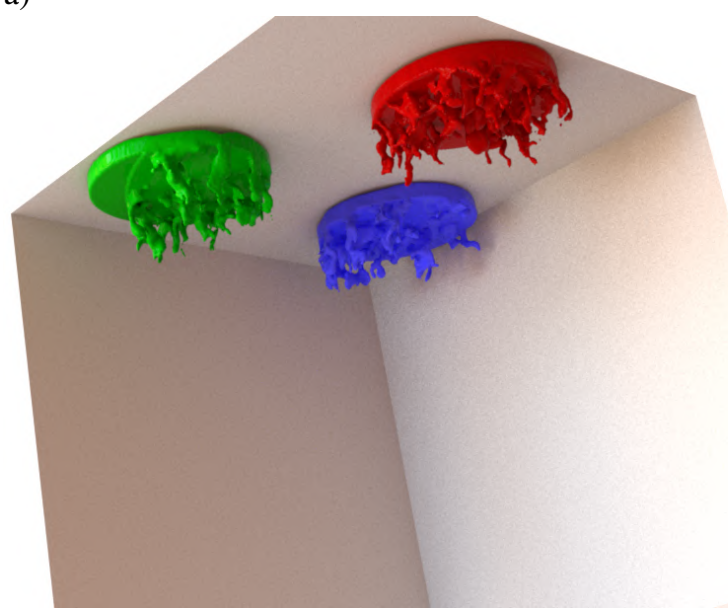

c)

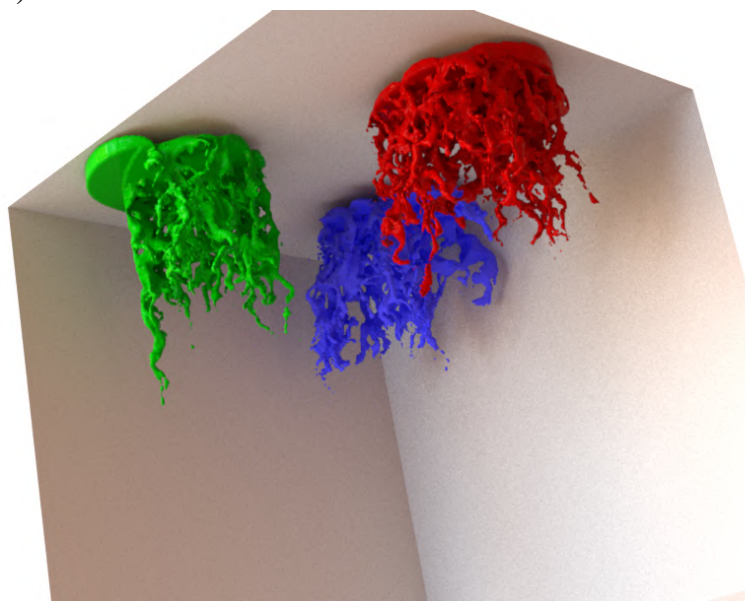

b)

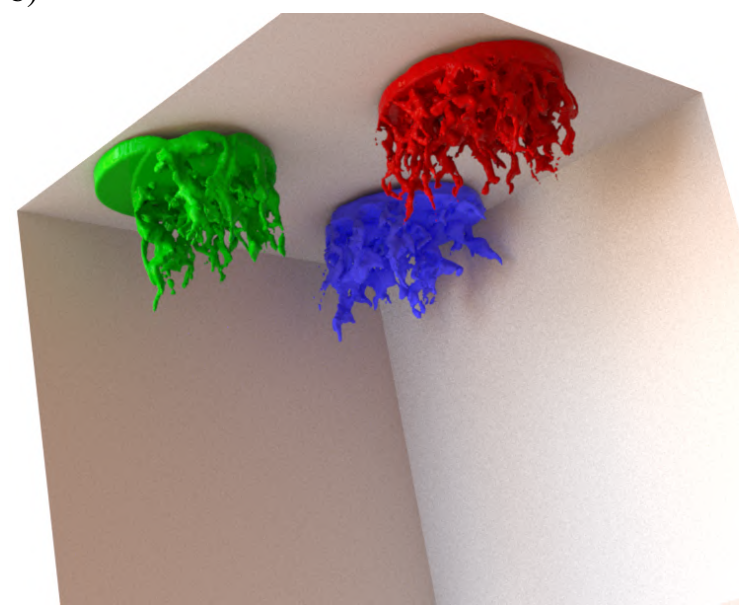

d)

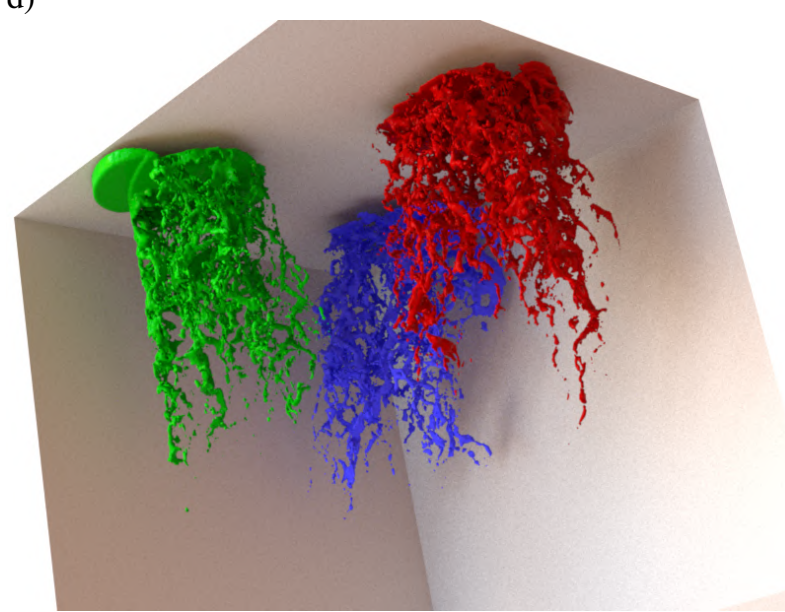

e)

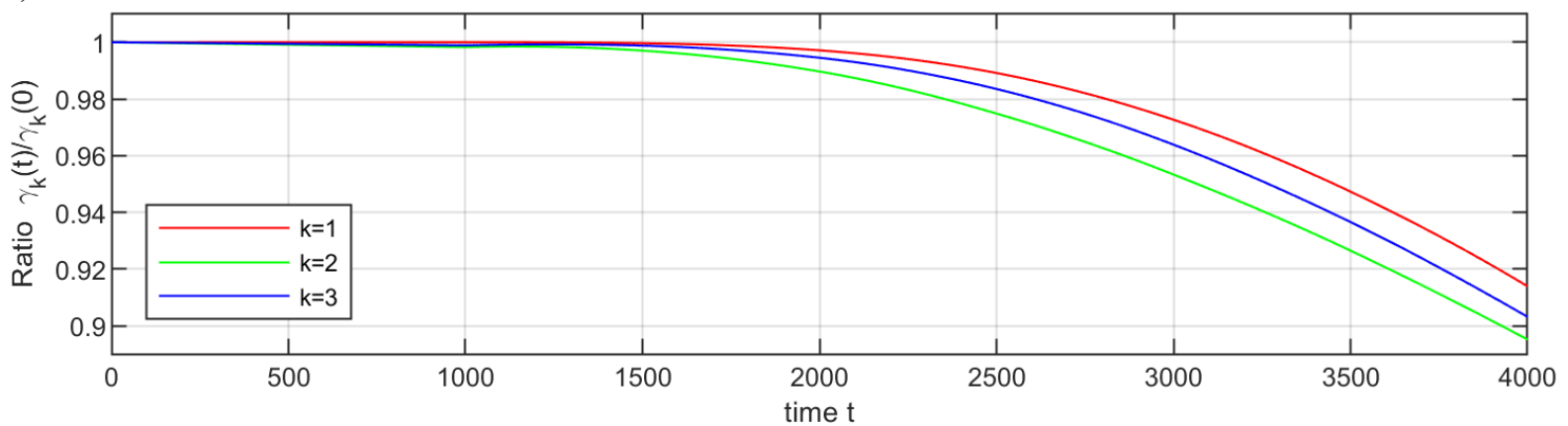

Figure 7: Transport of three passive scalars, initialized at one in three cylinders at the top the box, and zero elsewhere. The snapshots (a)-(d) show isovalues of the scalar fields after times $t=500,1000,2000$ and 4000 . The surrounding box is the unity box $[-1 / 2,1 / 2]^{3}$. Red, green and blue surfaces display respectively the first, second and third components of vector $C$. The curves on the plot (e) show the related balance values $\gamma_{k}(t) / \gamma_{k}(0)$ inside the pore space with respect to time.

Our velocity-vorticity formulation of the Stokes equation is quite far from vortex methods, but the particle formulation for transport phenomena is quite close.

Indeed, the particle formulation of the scalar field $C(x, t)$ is a set triplets $\left(x_{p}, C_{p}, v_{p}\right)$ indexed by $p$, where a particle of volume $v_{p}$ at position $x_{p}$ holds the quantity $C_{p} \in R^{3}$ (the sum of these elementary volumes is most of the time coinciding with the volume of the computational box). The equation (32) reads consequently, using the particle formulation:

$$
\frac{d C_{p}}{d t}=0, \frac{d x_{p}}{d t}=u\left(x_{p}(t)\right), \frac{d v_{p}}{d t}=0,
$$

where the velocity field $u$ is computed by the method introduced in the previous sections, and the dynamical systems satisfied by the passive scalars in their particle formulation are solved by a second-order Runge-Kutta method. The goal of this simulation is to show that the computed velocity field satisfies sufficiently accurately the condition $u \cdot n=0$ at the fluid/solid interface, so that the residual velocities at this interface don't allow particle to move into the solid. In 
order to do so, the figure 7 shows the ratio between the passive scalars $C_{k}$ inside the pores and its initial volume. This ratio reads $\gamma_{k}(t) / \gamma_{k}(0)$ using the following vector notation of $\gamma$

$$
\gamma(t)=\int_{\Omega} C(x, t)\left(1-\mathbf{1}_{S}(x)\right) d x=\sum_{p} C_{p}\left(1-\mathbf{1}_{S}\left(x_{p}\right)\right) \delta v_{p}
$$

and is supposed to stay close to 1 . Since the characteristic function of the solid is defined on grid points, its value on particles $\mathbf{1}_{S}\left(x_{p}\right)$ is estimated by means of a convolution between its grid-point values and the hat function kernel

$$
\Lambda^{\varepsilon}(x)=\varepsilon^{-3} \Lambda^{\otimes 3}(x / \varepsilon) \quad \text { with } \quad \Lambda(s)=[1-s]^{+}, s \in \mathbf{R},
$$

in order to deal with the lack of continuity of $\mathbf{1}_{S}$. Similarly, the velocity field is defined on the grid and has to be interpolated on the particles in order to evaluate $u\left(x_{p}(t)\right)$ in equation (33), here using the kernel $M_{4}^{\prime}$ introduced in [46] instead of the hat function thanks to a better continuity.

The figure 7 shows the evolution in the Castlegate sandstone of the three passive scalars, from their initial condition introduced above. We use the velocity field computed in the previous section 4.3. The simulation is performed over 4000 time steps with a constant time step $\delta t=1$. The figure shows that after 2000 time steps, the amount of particles moving from the pore space to the solid domain is around 1\%, and is around $10 \%$ after 4000 time steps. No remeshing is performed, in order to display how long the particles can move through the pores without going into the solid part due to numerical errors. This test is especially severe since all the particles have to follow complex paths as displayed on the plots (a)-(d) on figure 7.

\subsection{Upscaling and front invasion using a remeshed particle method}

In this section, we are interesting in upscaling the pore-scale configuration. Homogenization is the convergence toward a model involving the replication of cells whose size tends toward zero, and provides the proof that pore-scale Stokes homogenization is the Darcy law [5] in the context of stationary periodic solution of the homogenization. When the upscale solution is not periodic and subject to boundary condition, the Darcy law has to be generalized in order to take into account the variability in space. In the sections thereafter, we investigate this case, involving the concept of superficial velocity and the Darcy-Brinkman-Stokes model. Finally, the transport and diffusion in such an upcaled model is also investigated, providing methods and validity to the parameter estimation.

\subsubsection{The model of superficial velocity}

The upscaling of diffusion-transport is commonly performed using the formalism of the superficial velocity introduced by Quintard and Whitaker [56]. The superficial velocity $w$ is inherited from the pore-scale velocity $u$,in a representative cell $\Omega$ of volume $|\Omega|$ containing a fluid domain $\Omega_{f}$ of volume $\left|\Omega_{f}\right|$, by means of the relation

$$
w=\frac{1}{|\Omega|} \int_{V_{f}} u(x) \mathrm{d} x
$$

which can itself be variable in space, and satisfies

$$
-\phi^{-1} \mu \Delta w+\mu K^{-1} w=f-\nabla p
$$

in its stationary formulation at the Darcy scale $[72,69,11]$, together with $\operatorname{div} w=0$, where $\phi$ is the porosity of the medium and $K$ its permeability. Hence the need to provide estimations of porosity and permeability from pore-scale simulations in order to compute the superficial velocity. In the present study, one can get it by direct computation of integral (36).

One can notice that in the case of constant averaged velocity $w$, with a permeability estimated from a pore-scale computation using a non-porous (impermeable) solid domain $\Omega \backslash \Omega_{f}$, the equation (37) reduces to the definition of the permeability $\mu w=K f$ (the Darcy law), compatible with homogenization results [5].

Furthermore, the upscaling method mentioned above also involves the upscaled diffusion and transport of a specie $C$ (cf. $[56,63])$ by the equation

$$
\phi \frac{\partial C}{\partial t}+\operatorname{div}(w C)-\operatorname{div}(\phi \bar{\alpha}(\phi) \nabla(C))=0
$$

where $C$ is the intrinsic averaging of $C$ at the pore-scale defined by its mean value inside the fluid region

$$
C=\frac{1}{\left|\Omega_{f}\right|} \int_{\Omega_{f}} C(x) \mathrm{d} x .
$$

By means of the change of variable $\bar{C}=\phi C$, and assuming an upscaled porosity constant in time and space, the equation (38) can be written

$$
\frac{\partial \bar{C}}{\partial t}+\operatorname{div}\left(\phi^{-1} w \bar{C}\right)-\operatorname{div}\left(\bar{\alpha}(\phi) \nabla^{\phi} \bar{C}\right)=0
$$


a)

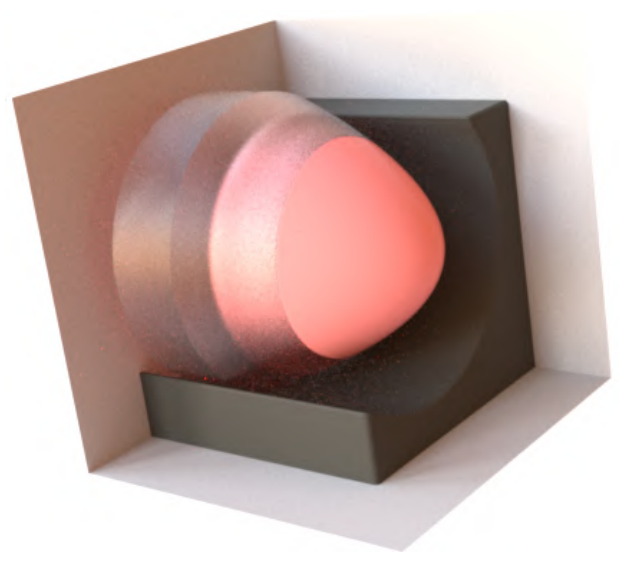

c)

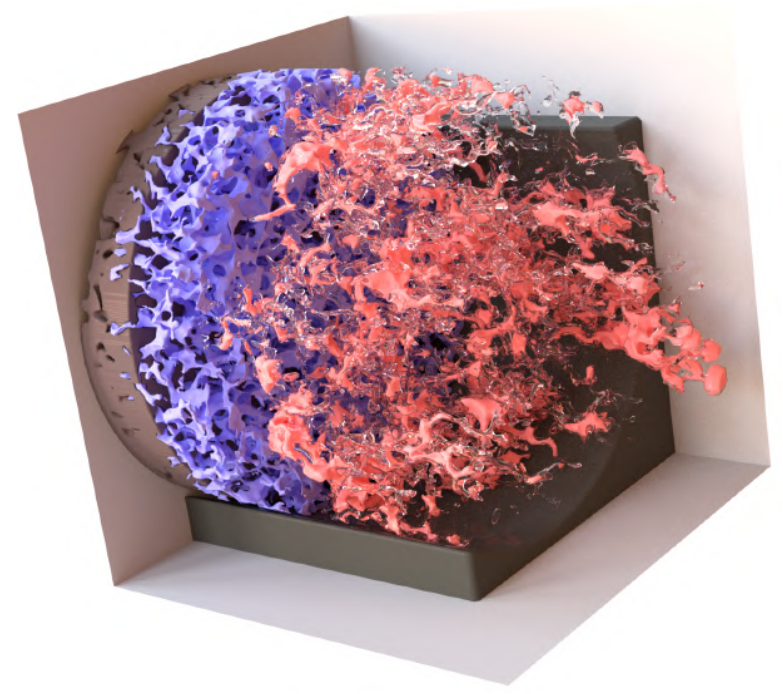

b)

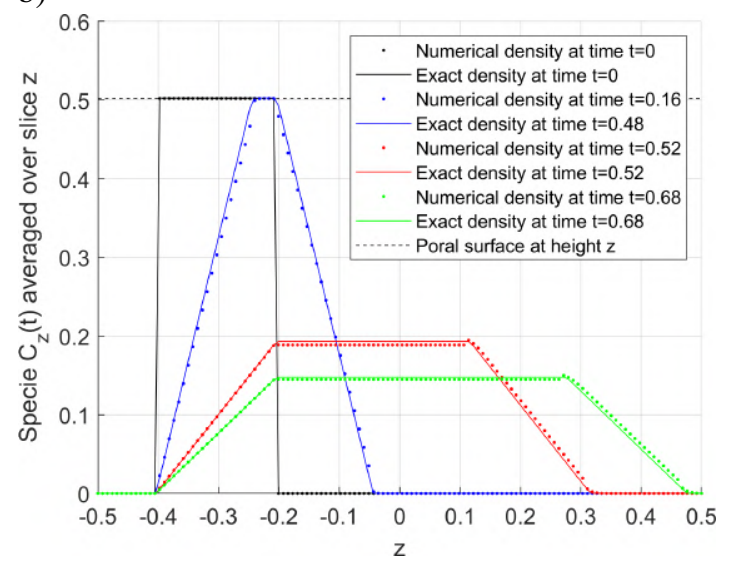

d)

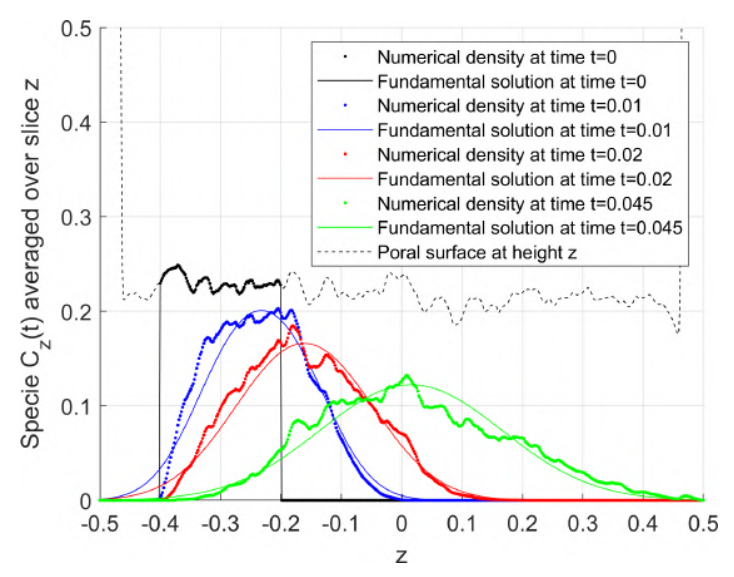

Figure 8: Upscaling to the model of averaged specie (38) and superficial velocity (37) for pure transport. Top pictures: Transport in a cylindrical pore of radius $0.4\left(128^{3}\right)$ with transparent color for radius over 0.28 and solid color under 0.28 (a); Comparison between theoretical (solid lines) and numercal (dots) distributions of $C$ at different times (b). Bottom pictures: Transport in a Castlegate sandstone $\left(512^{3}\right)$ with partial view of the cylindrical cell and the rock geometry (dark colors), initial value of $C$ in blue and after $t=0.05$ at level 0.8 in red and at level 0.5 in transparent (c); Comparison between 1D upscaled model using fundamental solutions with solid lines and numerical solutions plotted with dots (d). The planar faces delimit the unit computational box $[-1 / 2,1 / 2]^{3}$.

where $\nabla^{\phi}=\phi \nabla \phi^{-1}$ is the lifted gradient, equal to $\nabla$ since assuming the upscaled porosity to be constant.

This equation (38) has two versions of 1D models in relative coordinates: either the driving velocity $U=\phi^{-1} w$ is not zero, then we introduce the Peclet number $P e=U L / \bar{\alpha}$ and get

$$
\partial_{t} \bar{C}+\partial_{z} \bar{C}-P e^{-1} \Delta \bar{C}=0
$$

or the characteristic velocity is zero and we introduce the characteristic time $\alpha t / L^{2}$ and get $\partial_{t} \bar{C}-\Delta \bar{C}=0$.

The model (38) can be naturally generalized to reactive flows [45, 44], ion transport [6] or multi-phase flows [57]. This last equation is integrated numerically using the particle method introduced above, but using a remeshing procedure of particles at every time-step, using the hat function as kernel despite its low order, in order to avoid any spurious diffusion possibly introduced by remeshing and conserve the sign and the maximum principle on the variable $C$. the kernel $M_{4}^{\prime}$ is still used to interpolate the velocity from the grid to the particles.

In this upscaling method, in order to make the upscaled diffusion-transport (37)-(40) usable in practice, the following parameters have to be available:

- The porosity $\phi$, which is the proportion of fluid per unit of volume and is trivially estimated by direct counting of fluid voxels,

- The permeability $K$, which can be obtained by computing the velocity at the pore-scale, which has been performed above in section 4 ,

- The diffusion coefficient $\bar{\alpha}(\phi)$, depending on the material porosity, the molecular diffusion $D_{M}$ of the specie transported. 

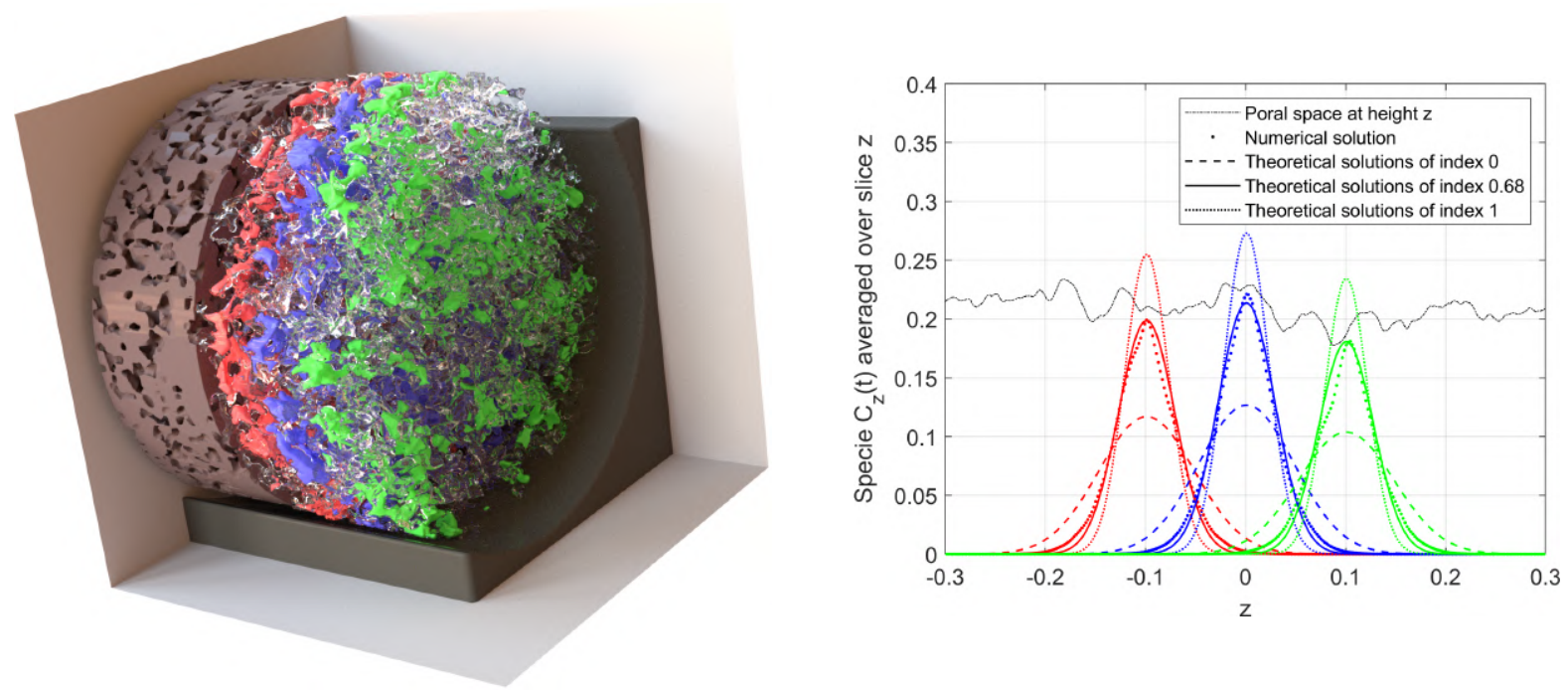

Figure 9: Upscaling the diffusion using the model of averaged specie (38). The evolution from three Dirac function at $z=-0.1,0$ and 0.1 are displayed at time $t=1.210^{-3}$ respectively in red, blue and green. The right pictures compares each of these numerical simulation at the pore-scale (in dots) to exact solution of the upscaled model for indexes $0,0.68$ and 1 . The left picture shows the isosurfaces of the pore-scale simulation at level 0.02 (solid colors) and 0.002 (transparent surface), at the same time and using the same colors as right picture.

The question addressed here is to use high resolution simulation at a scale such as it can represent both the porescale and the upscaled model in order to provide meaningful parameter estimations. The porosity and the permeability are direct estimations, but estimating and fitting the Archie law is more complex and requires the coupling between velocity field at the pore-scale and diffusion-transport, which is exactly the system (32).

\subsubsection{Upscaling pure transport in simple geometry}

In order to describe how to build the effective coefficients in the upscaled model of a porous structure, we will first consider the case of a cell perforated by a cylinder, for which we can build analytical solutions and explain the methodology (in this case, the material is a box with a single pore in relative coordinates). In a second time, we will apply this to a real geometry in the next section.

One then considers the non-dimensional Stokes flow in a cylinder of Radius $R<1$ of axis $z$ in a cubic unit computational box with full periodic boundary conditions (in relative coordinates), in which the Poiseuille flow is given in cylindrical coordinates by $u(r)=\left(1-r^{2} / R^{2}\right) U_{\max }$ along axis $z$ (its other components being zero) and satisfying $-\Delta u=4$. An obvious calculation gives that the averaged velocity in the fluid domain $\Omega_{f}$ is $U_{\operatorname{moy}}=U_{\max } / 2$, the porosity is the section $\phi=\pi R^{2}$ and the superficial/upscaled velocity is $w=\phi U_{\text {moy }}$.

Moreover, the displacement of a particle at a distance $r$ from the axis over a time $t$ is given by $\xi(r, t)=u(r) t$ so that the mean displacement of a section of the cylinder is given by $\bar{\xi}(t)=t U_{\text {moy }}$. The solution of a pure transport can then be expressed analytically.

Initially, let $C$ be equal to 1 in the circular pore at the axial location $z \in\left[z_{0}-H, z_{0}+H\right]$ with $z_{0}=-0.3$ and $H=0.1$. To make the link between the local values $C$ and the upscaled values $\bar{C}=\phi C$, let us follow its averaged value over the cylinder sections, quantified by

$$
C_{z}(t)=\frac{2 \pi}{S_{r e p}} \int_{0}^{R} C(r, z, t) r \mathrm{~d} r=\frac{\pi R^{2}}{S_{r e p}}\left(S_{2}(z, t)-S_{1}(z, t)\right)
$$

satisfying $C_{z}(0)=\pi R^{2}=\phi$ in the sections $z \in\left[z_{0}-H, z_{0}+H\right]$ and 0 everywhere else, with the representative section $S_{\text {rep }}=1$ being the whole slice of the unit computational box, and

$$
S_{2}(z, t)= \begin{cases}1 & \text { if } z \in\left[z_{0}-H, z_{0}+H\right], \\ 1-\frac{z-z_{0}-H}{U_{\text {max }} t} & \text { if } z \in\left[z_{0}+H, z_{0}+H+U_{\text {max }} t\right], \\ 0 & \text { otherwise, }\end{cases}
$$

and

$$
S_{1}(z, t)= \begin{cases}1-\frac{z-z_{0}+H}{U_{\max } t} & \text { if } z \in\left[z_{0}-H, z_{0}-H+U_{\text {max }} t\right], \\ 0 & \text { otherwise. }\end{cases}
$$

It follows that the variance of a section displaced by $\xi(r, t)$ is $t^{2} U_{m o y}^{2} / 3$ so the standard deviation reads $\sigma=$ $t \phi w / \sqrt{3}$. This implies several consequences. First, The upscaled model of pure transport should not be a model of 
pure transport. Nevertheless, this spreading is not related to diffusion, whose standard deviation scales as $t^{1 / 2}$ and not as $t$. This is illustrated by the numerical application for a radius $R=0.4$ in a computational box of size 1 in each direction at the resolution $128^{3}$, leading to $\phi \simeq 0.5$, with $U_{\max }=1$. The numerical and the exact evaluations of $C_{z}(t)$ are displayed on figure 8.b at several times, together with an isosurface of $C$ on figure 8.a. Second, this shows that when studying a front invasion by pore-scale simulations and its upscaling, it is useless or unphysical to fill the pores at the sample input, since only a part of it is transported significantly and reaches the adjacent cells since it travels mainly in the center of the pores. Third, the solution exhibits linear front and tail.

\subsubsection{Upscaling pure transport in a real sandstone}

We focus here on upscaling transport inside the Castlegate sandstone used and analysed in sections 4.3 and 5.1, in order to show that the effect of dispersion and linear front/tail mentioned above can be observed in practice, on real geometries. In this case, the material is a cylindrical representative sample, with multiple pores of complex geometry.

Let us consider again the Castlegate sandstone whose velocity field has been computed in section 4.3: in relative coordinates, the flow rate over the input face is $|\bar{u}|=1$ so that the superficial velocity averaged over the cylindrical cell is $w=1 / \pi R^{2}$ with $R=0.46$ and its porosity is $21.3 \%$. The simulation of this flow is described on figure $8 . c$, showing a part of the cylindrical cell and the rock sample, the initial condition of $C$ in blue with pores filled with 1 in the piece $z \in[-0.4,-0.2]$, and the resulting distribution of $C$ at $t=0.05$.

Moreover, the crosswise-averaged solution $C_{z}$ for this case is defined by

$$
C_{z}(t)=\frac{1}{S_{r e p}} \int_{\Omega_{F}} C(x, y, z, t) \mathrm{d} v
$$

where the representative section is the section of the rock sample $S_{\text {rep }}=\pi R^{2}$, the surrounding cell being ignored as non-relevant part of the material studied. This averaged quantity $C_{z}$ is displayed at several times on figure 8.d. As for the cylindrical pore. The numerical solution exhibits a linear queue since a portion of the fluid remains stuck in the boundary layer with a constant shear-rate, and the solution spreads so that the transported specie progressively exits the computational domain.

It is noticeable that the upscaled transport equation (40), seen as a $1 \mathrm{D}$ transport equation along the $z$ direction, has an analytical solution

$$
\bar{C}(z, t)=\left[\bar{C}_{0}\left(\cdot-\phi^{-1} w t\right) * G_{\alpha}\right](z)=\int_{\mathbf{R}} \bar{C}_{0}\left(z-y-\phi^{-1} w t\right) G_{\alpha}(y) \mathrm{d} y,
$$

that is to say the convolution between the initial value $\bar{C}_{0}=\bar{C}(\cdot, 0)$ and the $1 \mathrm{D}$ heat kernel

$$
G_{\alpha}(z)=\frac{1}{\sqrt{4 \pi \alpha t}} e^{-z^{2} / 4 \alpha t}
$$

with a velocity $\phi^{-1} w \simeq 7.06$. The figure 8 .d shows both $C_{z}(t)$ and $\bar{C}(z, t)$ in the equivalent medium. There is remarkable agreement between the mean positions, which validates the value of the superficial velocity $w$ and the part of the upscaled model related to the transport. Nevertheless, this simulation shows that it also comes with a relative diffusion $\bar{\alpha} \simeq 6.5$, that is to say a Peclet number $P e \simeq 0.154$ for the upscaled model, which is a highly diffusive. This dispersion/diffusion scaling may be quantified by the Bodenstein number typical of stirred-tank reactor [71].

Once again, the upscaling of transport by equation (38), or equivalently (40), is valid only if one considers the material circulating far from the pore boundaries instead of considering the whole pore section, which includes material stuck in the boundary layer. This has several consequences. Concerning multi-phase flows, the mean velocity is relevant since it is linked to the motion of the triple point and puts the material in motion in the whole pores, so that the model of transport with the superficial velocity is valid. Moreover, for single phase flows, only the viscous superficial model has to be considered, and a pure-transport version of model (38) should be avoided.Nevertheless, this theoretical question is not really a problem since practical configurations only involve highly diffusive fluids at this scale. In any case, such upscaling requires an accurate geometrical description of the poral space.

\subsubsection{Upscaling diffusion}

The question of upscaling pure diffusion (without driving velocity) is to link $\alpha$ at the pore scale to $\bar{\alpha}$ at the Darcy scale (40). In practice, the upscaled diffusion coefficient $\bar{\alpha}$ is usually expressed under the formulation

$$
\bar{\alpha}(\phi)=\phi^{m} D_{M}
$$

where $D_{M}=\alpha$ is the molecular diffusion at the pore scale. This is known as the Archie law [10], which has been tabulated empirically for decades [70]. For moderately porous media with strong pore connections such as sedimented rocks, sandstones or crystal wormholing, the model $\bar{\alpha}=\phi D_{M}$ (with an index $m=1$ ) is often considered (cf. [63] or [70] for bead stacks). 

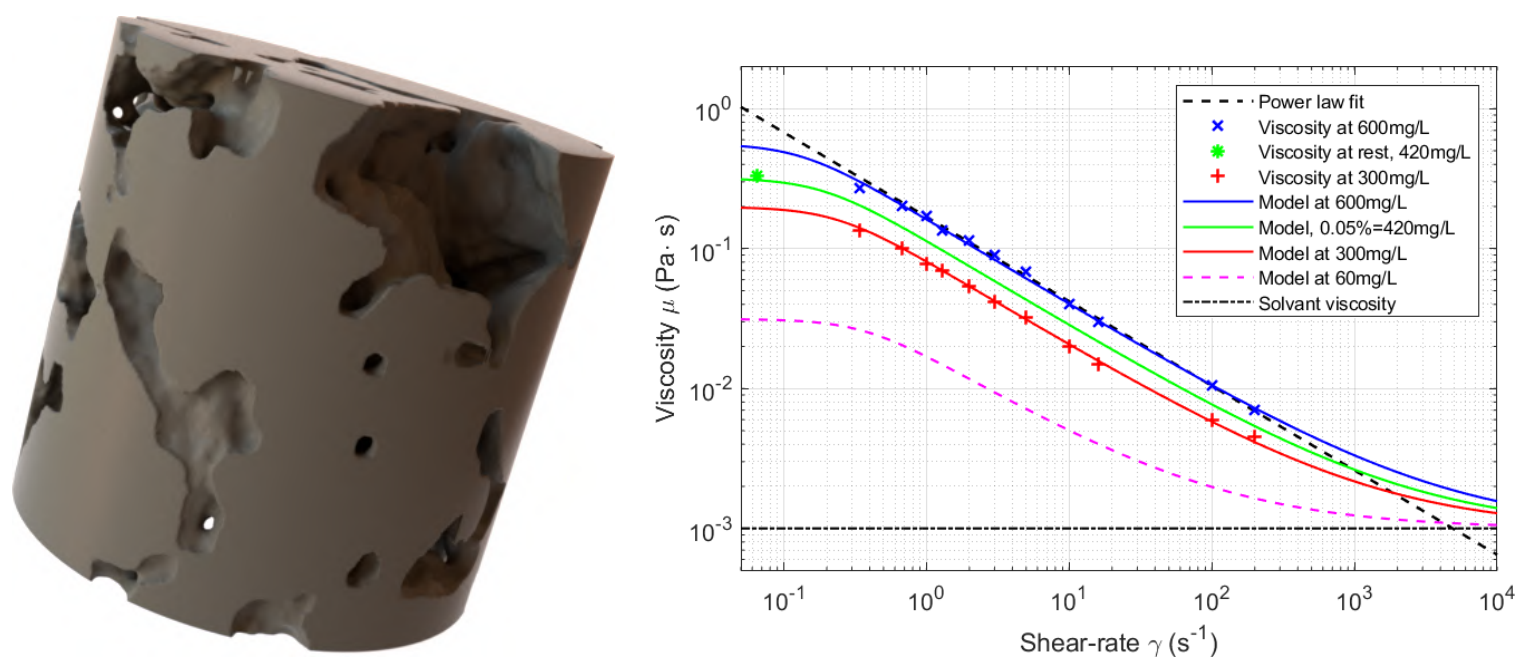

Figure 10: To the left: the body used for simulation, the full solid region being this body and the complementary of the cylindrical region, surrounding this body and filling the computational box. To the right: Rheogram showing the dependency of viscosity with respect to the shear rate for different Xanthan concentrations, with experimental values (points) and their fit (lines). Solvent viscosity and power-law approximation are displayed with black lines. Experimental data and their fit to the Carreau law are taken from [60].

Furthermore, as mentioned above, from now on we use the non-dimensional time in the simulations defined by $\alpha t / L^{2}$. In order to perform this upscaling of diffusion, we use a numerical simulation inspired from a technique used in chemical engineering: we set as initial condition the Dirac function hold by a slice $z=z_{0}$ defined by the generalized function

$$
\langle C(\cdot, t=0), \zeta\rangle=\int_{z=z_{0}} A \zeta(x, y, z)\left(1-\mathbf{1}_{S}(x, y, z)\right) \mathrm{d} x \mathrm{~d} y
$$

with $A>0$. It follows that the initial upscaled is supposed to be $\bar{C}(z, t=0)=A \phi_{z} \delta_{z_{0}}$ where $\phi_{z}$ is the porosity of the slice $z=z_{0}$, so that we expect the solution

$$
\bar{C}(z, t)=A \phi_{z} G_{\phi^{m}}(z, t)
$$

in relative coordinates.

To perform these simulations, the Laplace operator is computed using a renormalized particle-strength-exchange introduced in [53,62], intrinsically converging a second order of the grid step size, that is to say independently from the size of the diffusion kernel. Its main advantages are that it can easily and strictly avoid diffusion inside the solid and provide an accurate computation of diffusion inside the poral space.

The simulations with $A=0.1$ and $z_{0}=-0.1,0$ and 0.1 have been performed and are displayed on figure 9 at the relative time $t=1.210^{-3}$, respectively in red, blue and green. Each of these solutions at the pore scale are compared to the theoretical solutions (46) with several indexes. By comparison, the molecular diffusion of hydrochloric acid in water is $510^{-5} \mathrm{~cm}^{2} \mathrm{~s}^{-1}$ [44], with a characteristic length $L=1.8 \mathrm{~mm}$ for the present sample (see section 4.3 ), so that the relative time $t=1.210^{-3}$ leads to an absolute time $t=0.78 \mathrm{~s}$. It appears that for this geometry the index $m=0.68$ fits well to the numerical solutions. These simulations confirm that the straightforward copy of the molecular diffusion (i.e. $m=0$ ) in the upscaled model is wrong, and that the linear Archie law (i.e. $m=1$ ) used in [63] or [70] for bead stacks is over-diffusive.

To conclude, the present method used for pore-scale simulation allows to build the diffusion coefficient in the upscaled model (38), here an index $m=0.68$ in the Archie law (45).

\subsection{Transport of active scalar for a fully coupled model involving rheology}

In this section we are interested in the rheology of shear-thinning fluids, here modeled by the Carreau-law, a power-law with two bounds featuring the Newtonian limits at high and low shear rates, defined by

$$
\mu(D)=\mu_{\infty}+\left(\mu_{0}-\mu_{\infty}\right)\left(1+2 \beta^{2}|D|^{2}\right)^{(N-1) / 2},
$$

where $\mu_{0}$ is the viscosity at rest and $\mu_{\infty}$ is the asymptotic value of the viscosity at infinite shear-rate, in practice the viscosity of the solvent, satisfying $0<\mu_{\infty} \leqslant \mu_{0}$. The shear rate threshold $\beta$ quantifies the shear rate for which the viscosity becomes dramatically different from $\mu_{\infty}$, and $N$ is the called the index of the fluid. The scalar shear rate is defined by $\dot{\gamma}=(2 D(u): D(u))^{1 / 2}$. Due to the focus on a real configuration in this section, we express all the quantities in their dimensional form with units (that is to say in absolute coordinates).

Such a law is often associated to the dynamics of polymers or bio-polymers. Real-life configurations naturally involve heterogeneous fluids, that is to says fluids whose rheology depends on the local concentration of the polymer 

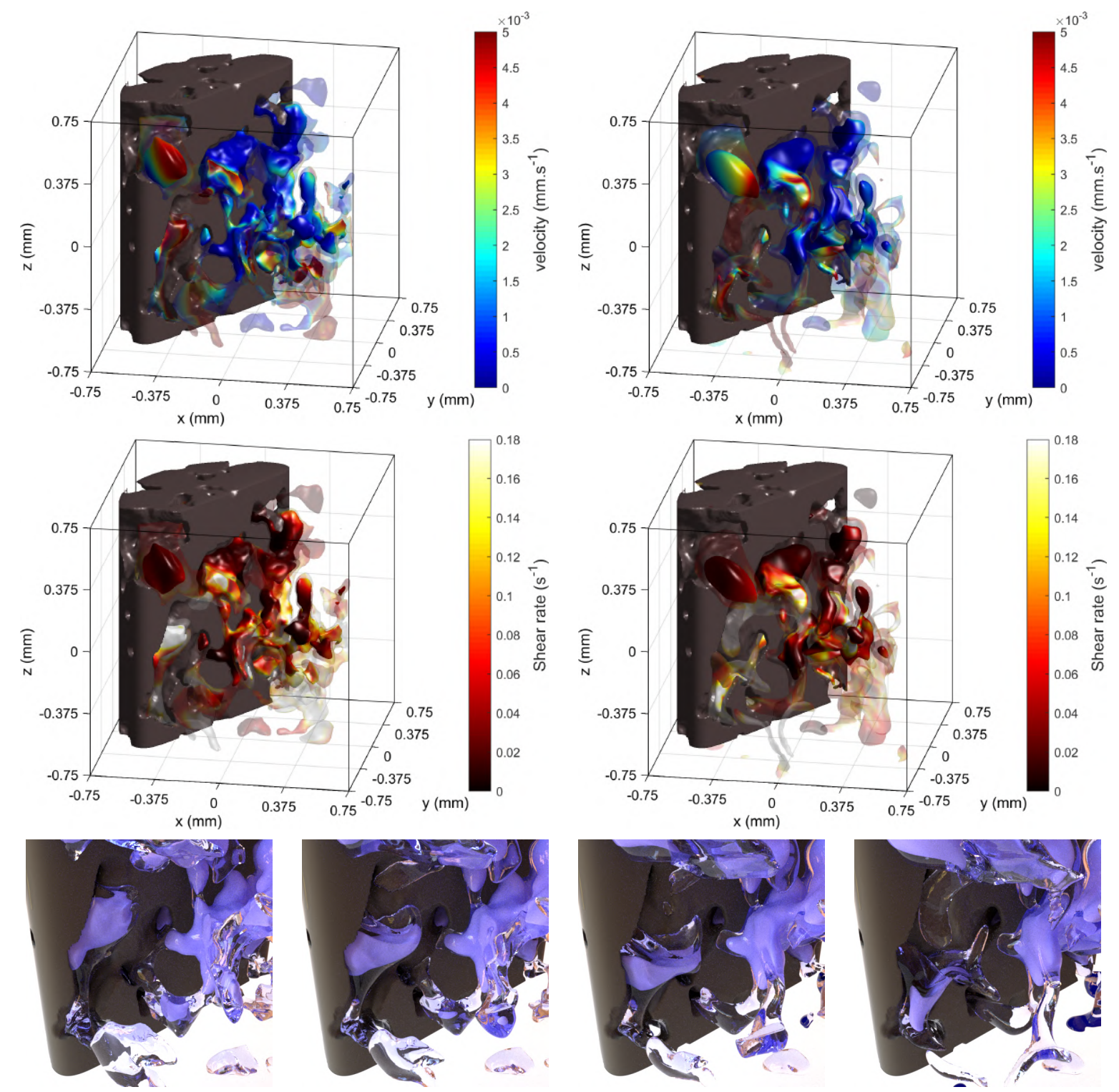

Figure 11: Four top pictures: Isosurfaces of Xanthan concentration at levels $C / \bar{C}=0.3$ (solid color) and 0.1 (transparent color). This shows its evolution between the initial time (left pictures) and $t=64 \mathrm{~s}$ (right pictures), colored by velocity (top pictures) and shear-rate (bottom pictures). The four bottom pictures display a rendering of this evolution by means of a zoom on a transport-dominant region after $0,16,24$ and 32 time steps.

considered. This implies a relation between $\mu$ and $C$ in the set of equations (32). The scalar $C$ becomes consequently an active tracer by means of the Carreau law (47) generalized to variable parameters, which leads to the following set of equations:

$$
\left\{\begin{array}{l}
\frac{\partial C}{\partial t}+\operatorname{div}(u \otimes C)-\alpha \Delta C=0 \\
-\operatorname{div}(2 \mu(C, D(u)) D(u))+\nabla p+\frac{\mathbf{1}_{S}}{\varepsilon}(u-\bar{u})=0 \\
\mu(C, D)=\mu_{\infty}+\left(\mu_{0}(C)-\mu_{\infty}\right)\left(1+2 \beta(C)^{2}|D|^{2}\right)^{(N(C)-1) / 2} \\
\operatorname{div} u=0
\end{array}\right.
$$

with

$$
\operatorname{div}(2 \mu(C, D(u)) D(u))=\mu(C, D(u)) \Delta u+2 D(u) \nabla \mu(C, D(u))
$$

since $\operatorname{div} u=0$, the term $D \nabla \mu$ being used at the right hand side as a source in the algorithm developed above.

We consider the body defined by a cylindrical core of Bentheimer rock displayed on figure 10. This cylindrical core of radius $R=0.71 \mathrm{~mm}$ is extended radially by a carter in order to fill the cubic computational box of width $L=1.5 \mathrm{~mm}$ : the body and the carter define the penalized solid region $S$.

The application considered in the present work is the motion of a miscible agglomerate of Xanthan polymer, a bio-polymer heavy used in food industry and also in enhanced oil recovery, featuring a constant index $N=0.386$ and

$$
\mu_{0}(C)=\mu_{\infty}+A_{\mu} e^{C / \rho B_{\mu}}\left(1-e^{-C / \rho R_{\mu}}\right), \quad \beta(C)=A_{\beta} e^{C / \rho B_{\beta}},
$$



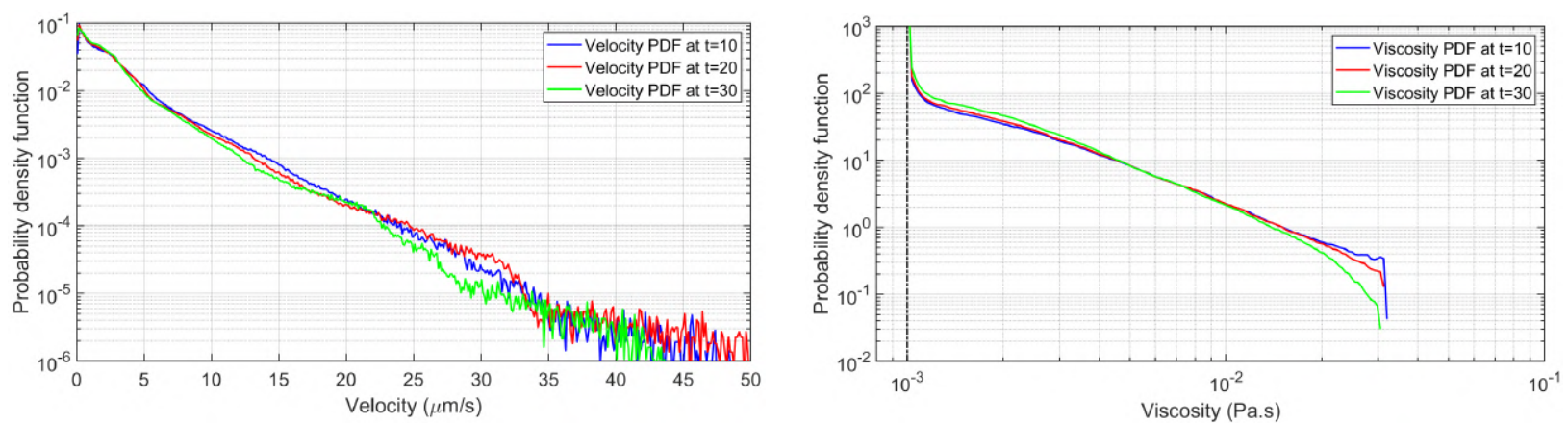

Figure 12: Probability density functions of the velocity field (to the left) and the viscosity field (to the right), in the fluid domain, at three different times. The dashed line stands for the solvent viscosity (Newtonian limit).

where $\rho=0.842 \mathrm{~kg} \mathrm{~L}^{-1}$ is the density of the pure material, the nominal time is $A_{\beta}=2.4 \mathrm{~s}$, the nominal and solvent viscosity are $A_{\mu}=0.26 \mathrm{~Pa}$ s and $\mu_{\infty}=10^{-3} \mathrm{~Pa}$ s (water), the non-dimensional ratio are $B_{\mu}=6.0610^{-4}, R_{\mu}=6.510^{-4}$ and $B_{\beta}=6.1710^{-4}$ (see [60] for instance). This leads to the rheogram, that is to say the relations between the viscosity and the shear rate, displayed on figure 10 .

Furthermore, the initial condition on $C$ is a random field between 0 and $\bar{C}=600 \mathrm{mg} \mathrm{L}^{-1}$, generated by a FFTMA algorithm (FFT Moving Average, [59]) with a correlation length of $0.146 \mathrm{~mm}$ [48] and supported by the fluid region. The driving velocity is $\bar{u}=510^{-4} \mathrm{mms}^{-1}$, the diffusion is $\alpha=410^{-12} \mathrm{~m}^{2} \mathrm{~s}^{-1}$, the computational grid is 256 cells in each direction and the time step is $\delta t=2 \mathrm{~s}$. Moreover, the kernels used are $M_{4}^{\prime}$ for the velocity interpolation and $M_{3}$ for the concentration interpolation [46].

The results of this simulation is display on figure 11 for the initial time and after 32 time steps, by means of surfaces of isovalue of $C / \bar{C}$ at levels 0.3 and 0.1 , as well as a hollow plot of the body. Both velocity and shear rate are displayed, and since the concentration is constant over these surfaces, a lower shear rate means a higher viscosity (because of the fluid index $N<1$ leading to a shear-thinning effect).

Beside illustrating the robustness of the method (viscosity varies by 3 orders of magnitude), one can notice on this figure 11 that some regions are driven by transport (red velocity and white/yellow shear rate) while others are driven by diffusion (blue velocity and dark-red shear rate). The bottom left part exhibits a region where both velocity and shear-rate are high, with large variations: the evolution of the Xanthan concentration at several times is also displayed at the bottom part of this figure 11 .

Concerning physics aspects, we can quantify the velocity and viscosity distributions, displayed on the figure 12 by means of the probability density functions (PDF) of these fields. It appears that the velocity PDF exhibits an exponential law, and that the viscosity field exhibits a rational decay. Moreover, the asymptotic most probable value fits to the solvent viscosity $\mu_{\infty}$. This shows that most of the fluid is either low concentration Xanthan or high shear rate, but the maximum viscosity reached by the fluid is 30 times higher (as well as its related stability condition).

\section{Conclusions and perspectives}

In order to compute the flow at the pore scale of porous media, we have considered a time-dependent version of Stokes equation to build an iterative method. By the use of the velocity and the vorticity fields, the different operators (penalization, diffusion, projection) have been treated separately thanks to a splitting method. Each step only requires direct computation or a call to a FFT solver. The resulting algorithm has been shown to be consistent with the problem to solve, and corresponds to another penalization whose coefficient depends on the initial penalization coefficient and chosen time step.

The numerical implementation of this algorithm has been performed on three examples, including two real world cases consisting in X-Ray scans of stone and bead stacks. For each sample, the convergence has been confirmed by conservation and residual quantities, such as the residual velocity in the solid part of the domain, and permeability estimations. A coupling with material transport through the pores has been performed in order to evaluate the quality of the resulting velocity field for transport process. This method has also been used for parameter estimation in upscaling to the Darcy scale. While upscaling diffusion seems to be reliable by fitting the index of an Archie law, the concept of superficial velocity may be adapted in the context of pure-transport of one-phase flow, due to the effect of boundary layer, and does not need to be adapted for multi-phase or diffusion-driven flows.

The robust method developed in this paper can be a self-content component dedicated to the computation of the velocity inside coupled problems, in the field of reactive flows, multi-scale porosity media and multi-phase flows at low Reynolds regimes, complex fluid with rheology or plasticity, or fluid-solid interaction, strength of porous materials along with their study up to the failure. 


\section{Acknowledgments}

We gratefully acknowledge the help of Pr P. Moonen from D-MEX, UMS 3360 UPPA-CNRS-Total for the microCT scan of beads stacks. This work has been partially supported by the Conseil Départemental des Pyrénées-Atlantiques by means of Grant 2015-0769 and by the Carnot Institute ISIFoR by means of Grant RugoRX P450704ISI. This work was granted access to the HPC resources of Pyrene (UPPA Cluster, France) and Curta (University of Bordeaux, France).

\section{Appendix A. Consistency of the splitting of affine operators.}

Let us consider first an evolution equation $\partial_{t} u-A u-B u=0$ over a time interval $[0, \delta t]$ whose initial condition is denoted $u_{0}$ and exact solution is $u(t)=e^{(A+B) t} u_{0}$. The operator $A+B$ can then be treated with an operator splitting, which means dealing separately with $A$ and $B$. This splitting consists in solving sequentially the two equations

$$
\left\{\begin{array}{l}
\frac{\partial u_{1}}{\partial t}-A u_{1}=0 \\
\frac{\partial u_{2}}{\partial t}-B u_{2}=0
\end{array}\right.
$$

whose initial conditions are $u_{1}(0)=u_{0}$ and $u_{2}(0)=u_{1}(\delta t)=e^{A \delta t} u_{0}$.

It follows that the truncation error over a time step $[0, \delta t]$ is given by

$$
u(\delta t)-u_{2}(\delta t)=e^{(A+B) t} u_{0}-e^{B \delta t} e^{A \delta t} u_{0}=\frac{\delta t^{2}}{2}[A, B] u_{0}+O\left(\delta t^{3}\right)=O\left(\delta t^{2}\right)
$$

with $[A, B]=A B-B A \neq 0$ in the case of a priori non-commuting operators $A$ and $B$, since $e^{z}=\sum_{j=0}^{\infty} \frac{z^{k}}{k !}$.

This means a global first order error induced by the splitting, error which can be reduced to second order with a Strang splitting formula [66].

In the present article, we are interested in affine operators defining the equation

$$
\frac{\partial u}{\partial t}-A u-B u=C
$$

whose solution is

$$
u(t)=e^{(A+B) t} u_{0}+(A+B)^{-1}\left(e^{(A+B) t}-1\right) C=e^{(A+B) t} u_{0}+t \varphi_{1}((A+B) t) C,
$$

where

$$
\varphi_{1}(z)=z^{-1}\left(e^{z}-1\right)=\sum_{j=0}^{\infty} \frac{z^{k}}{(k+1) !}
$$

is the function classically introduced in the numerical analysis of exponential integrators, satisfying $\lim _{z \rightarrow 0} \varphi_{1}(z)=1$.

The splitting between diffusion on the one hand and penalization-pressure on the other hand is written

$$
\left\{\begin{array}{l}
\frac{\partial u_{1}}{\partial t}-A u_{1}=0, \\
\frac{\partial u_{2}}{\partial t}-B u_{2}=C,
\end{array}\right.
$$

whose initial conditions are also $u_{1}(0)=u_{0}$ and $u_{2}(0)=u_{1}(\delta t)=e^{A \delta t} u_{0}$. One gets immediately

$$
u_{2}(t)=e^{B t} u_{2}(0)+t \varphi_{1}(B t) C=e^{B t} e^{A t} u_{0}+t \varphi_{1}(B t) C
$$

so that the truncation error is given by

$$
\begin{aligned}
u(\delta t)-u_{2}(\delta t) & =e^{(A+B) t} u_{0}-e^{B \delta t} e^{A \delta t} u_{0}+\delta t\left[\varphi_{1}((A+B) \delta t)-\varphi_{1}(B \delta t)\right] C \\
& =\frac{\delta t^{2}}{2}[A, B] u_{0}+\frac{\delta t}{2}\left[(A+B) \delta t-B \delta t+O\left(\delta t^{2}\right)\right] C+O\left(\delta t^{3}\right) \\
& =\frac{\delta t^{2}}{2}\left([A, B] u_{0}+A C\right)+O\left(\delta t^{3}\right)=O\left(\delta t^{2}\right)
\end{aligned}
$$

It is noticeable that in this case, the global error is still first order even if operators $A$ and $B$ commute or if a Strang algorithm is used. 


\section{References}

[1] J.C. Adams. MUDPACK-2: Multigrid software for approximating elliptic partial differential equations on uniform grids with any resolution. Applied Mathematics and Computation, 53(2):235 - 249, 1993.

[2] R. A. Adams and J. J. F. Fournier. Sobolev Spaces. Academic Press, June 2003.

[3] L.T. Akanji and S.K. Matthai. Finite element-based characterization of pore-scale geometry and its impact on fluid flow. Transp. Porous Med., 81:241-259, 2010.

[4] A.M. Alhammadi, A. AlRatrout, B. Bijeljic, and M.J. Blunt. Pore-scale imaging and characterization of hydrocarbon reservoir rock wettability at subsurface conditions using X-ray microtomography. Journal of Visualized Experiments, 2018.

[5] G. Allaire. Homogenization and two-scale convergence. SIAM J. Math. Anal., 23(6):1482-1518, 1992.

[6] G. Allaire, R. Brizzi, J. Dufrêche, A. Mikelić, and A. Piatnitski. Ion transport in porous media: derivation of the macroscopic equations using upscaling and properties of the effective coefficients. Comput. Geosci., 17:479-495, 2013.

[7] C. Anderson and C. Greengard. On vortex methods. SIAM Journal on Numerical Analysis, 22(3):413-440, June 1985.

[8] P. Angot, C.-H. Bruneau, and P. Fabrie. A penalization method to take into account obstacles in incompressible viscous flows. Numerische Mathematik, 81(4):497-520, 1999.

[9] P. Angot, G. Carbou, and V. Péron. Asymptotic study for Stokes-Brinkman model with jump embedded transmission conditions. Asymptotic Analysis, 2015

[10] G. E. Archie. The electrical resistivity log as an aid in determining some reservoir characteristics. Petroleum Transactions of AIME, 146:54$62,1942$.

[11] M.T. Balhoff, S.G. Thomas, and M.F. Wheeler. Mortar coupling and upscaling of pore-scale models. Comput. Geosci., 12:15-27, 2008.

[12] P. Barreau, D. Lasseux, H. Berlin, and A. Zaitowf. Effect of adsorbed polymers on relative permeability and capillary pressure: a pore scale numerical study. WIT Transactions on Modelling and Simulation, 10, 1970.

[13] J. T. Beale and A. Majda. Rates of convergence for viscous splitting of the navier-stokes equations. Mathematics of Computation, 37(156):243-259, October 1981.

[14] M. Bergmann and A. Iollo. Modeling and simulation of fish-like swimming. J. Comput. Phys., 230:329-348, 2011.

[15] I.I. Bogdanov, F. Guerton, J. Kpahou, and A.M. Kamp. Direct pore-scale modeling of two-phase flow through natural media. COMSOL Conference (Stuttgart), 2011.

[16] C. Cancès, T. Gallouët, M. Laborde, and L. Monsaingeon. Simulation of multiphase porous media flows with minimising movement and finite volume schemes. European Journal of Applied Mathematics, 30(6):1123-1152, 2019.

[17] G. Carbou. Brinkmann model and double penalization method for the flow around a porous thin layer. Journal of Mathematical Fluid Mechanics, 10(1):126-158, 2008

[18] G. Carbou and P. Fabrie. Boundary layer for a penalization method for viscous incompressible flow. Advances in Differential Equations, 8:1453-1480, 2003.

[19] P. C. Carman. Fluid flow through granular beds. Transactions, Institution of Chemical Engineers, London, 15:150-166, 1937.

[20] P. Chatelain, A. Curioni, M. Bergdorf, D. Rossinelli, W. Andreoni, and P. Koumoutsakos. Billion vortex particle direct numerical simulations of aircraft wakes. Computer Methods in Applied Mechanics and Engineering, 197:1296-1304, 2008.

[21] R. Chatelin, D. Sanchez, and P. Poncet. Analysis of the penalized 3D variable viscosity Stokes equations coupled to diffusion and transport. ESAIM: Mathematical Modelling and Numerical Analysis, 50:565-591, 2016.

[22] Z. Chen, G. Huan, and Y. Ma. Computational Methods for Multiphase Flows in Porous Media. SIAM, 2006.

[23] M. Cisternino and L. Weynans. A parallel second order Cartesian method for elliptic interface problems. Communications in Computational Physics, 12:1562 - 1587, 2012.

[24] G.-H. Cottet and P. D. Koumoutsakos. Vortex Methods: Theory and Practice. Cambridge University Press, March 2000.

[25] B. Couet, O. Buneman, and A. Leonard. Simulation of three-dimensional incompressible flows with a vortex-in-cell method. Journal of Computational Physics, 39(2):305-328, February 1981.

[26] M. El Ossmani and P. Poncet. Efficiency of multiscale hybrid grid-particle vortex methods. Multiscale Modeling $\mathcal{E}$ Simulation, 8(5):16711690, January 2010.

[27] J-M. Etancelin, P. Moonen and P. Poncet. Improvement of remeshed Lagrangian methods for the simulation of dissolution processes at pore-scale. To appear in Advances in Water Resources, 2020. https://doi.org/10.1016/j.advwatres.2020.103780

[28] I. Farago. A modified iterated operator splitting method. Applied Mathematical Modelling, 32(8):1542-1551, 2008.

[29] M. Gazzola, P. Chatelain, Wim M. van Rees, and P. Koumoutsakos. Simulations of single and multiple swimmers with non-divergence free deforming geometries. Journal of Computational Physics, 230(19):7093-7114, August 2011.

[30] T. Gillis, G. Winckelmans, and P. Chatelain. An efficient iterative penalization method using recycled krylov subspaces and its application to impulsively started flows. Journal of Computational Physics, 347:490 - 505, 2017.

[31] M.M. Hejlesen, P. Koumoutsakos, A. Leonard, and J.H. Walther. Iterative Brinkman penalization for remeshed vortex methods. Journal of Computational Physics, 280:547-562, 2015.

[32] U. Hornung, editor. Homogenization and Porous Media, volume 6. Interdisciplinary Applied Mathematics Series - Springer Verlag, 1997.

[33] L. Hume. Approche numérique d'écoulements complexes en milieu poreux à l'échelle des pores. PhD thesis, Université de Pau et des Pays de l'Adour, 2018

[34] L. Hume, F. Guerton, P. Moonen, and P. Poncet. Experimental and numerical cross-validation of flow in real porous media, Part 2: Numerical framework. 3rd International Conference on Tomography of Materials and Structures, 2017.

[35] W.H. Hundsdorfer and J.G. Verwer. Numerical solution of time-dependent advection-diffusion-reaction equations. Springer, 2003.

[36] S. Khirevich and T. W. Patzek. Behavior of numerical error in pore-scale lattice boltzmann simulations with simple bounce-back rule: Analysis and highly accurate extrapolation. Physics of Fluids, 30(9):093604, 2018.

[37] P. Koumoutsakos. Multiscale flow simulations using particles. Annual Review of Fluid Mechanics, 37(1):457-487, 2005.

[38] J. Kozeny. Ueber kapillare leitung des wassers im boden. Sitzungsber Akad. Wiss., Wien, 136(2a):271-306, 1927.

[39] L.W. Lake. Enhanced Oil Recovery. Prentice Hall, 1989.

[40] S. Liska and T. Colonius. A fast lattice green's function method for solving viscous incompressible flows on unbounded domains. Journal of Computational Physics, 316:360 - 384, 2016.

[41] B. Maury. Numerical analysis of a finite element/volume penalty method. SIAM Journal on Numerical Analysis, 47:1126-1148, 2009.

[42] C. Mimeau, G.-H. Cottet, and I. Mortazavi. Direct numerical simulations of three-dimensional flows past obstacles with a vortex penalization method. Computers $\mathcal{E}$ Fluids, 136:331 - 347, 2016.

[43] H.K. Moffatt. The degree of knottedness of tangled vortex lines. Journal of Fluid Mechanics, 35(1):117-129, 1969.

[44] S. Molins, C. Soulaine, N. I. Prasianakis, A. Abbasi, P. Poncet, A. J.C. Ladd, V. Starchenko, S. Roman, D. Trebotich, H. A. Tchelepi, and C. I. Steefel. Simulation of mineral dissolution at the pore scale with evolving fluid-solid interfaces: Review of approaches and benchmark problem set. Computational Geosciences, 2020. 
[45] S. Molins, D. Trebotich, C. I. Steefel, and C. Shen. An investigation of the effect of pore scale flow on average geochemical reaction rates using direct numerical simulation. Water Resources Research, 48(3), 2012.

[46] J.J Monaghan. Extrapolating b splines for interpolation. Journal of Computational Physics, 60(2):253-262, 1985.

[47] Y. Morinishi, T. S. Lund, O. V. Vasilyev, and P. Moin. Fully conservative higher order finite difference schemes for incompressible flow. Journal of Computational Physics, 143(1):90-124, 1998.

[48] B. Noetinger, L. Hume, R. Chatelin, and P. Poncet. Effective viscosity of a random mixture of fluids. Phys. Rev. Fluids, 3:014103, Jan 2018.

[49] A. T. Patera. A spectral element method for fluid dynamics: Laminar flow in a channel expansion. Journal of Computational Physics, 54(3):468 - 488, 1984.

[50] J.P. Pereira-Nunes, M.J. Blunt, and B. Bijeljic. Pore-scale simulation of carbonate dissolution in micro-CT images. J. Geophys. Res. Solid Earth, 121:558-576, 2016.

[51] P Ploumhans and G.S Winckelmans. Vortex methods for high-resolution simulations of viscous flow past bluff bodies of general geometry. Journal of Computational Physics, 165(2):354 - 406, 2000.

[52] P. Poncet. Topological aspects of three-dimensional wakes behind rotary oscillating cylinders. Journal of Fluid Mechanics, 517:27-53, 2004.

[53] P. Poncet. Finite difference stencils based on particle strength exchange schemes for improvement of vortex methods. Journal of Turbulence, 7:N23, January 2006

[54] P. Poncet. Analysis of direct three-dimensional parabolic panel methods. SIAM Journal on Numerical Analysis, 45(6):2259-2297, 2007.

[55] M. Quintard and S. Whitaker. Ecoulement monophasique en milieu poreux : Effet des hétérogénéités locales. Journal de Mécanique théorique et appliquée, 6:691-726, 1987.

[56] M. Quintard and S. Whitaker. Two phase flow in heterogeneous porous media: the method of large-scale averaging. Transport in Porous Media, 3:357-413, 1987

[57] M. J Raeini, A. Q. and. Blunt and B. Bijeljic. Direct simulations of two-phase flow on micro-ct images of porous media and upscaling of pore-scale forces. Advances in Water Resources, 74:116 - 126, 2014.

[58] J.T. Rasmussen, G.-H. Cottet, and J. H. Walther. A multiresolution remeshed vortex-in-cell algorithm using patches. Journal of Computational Physics, 230(17):6742-6755, July 2011.

[59] M.L. Ravalec, B. Noetinger, and L.Y. Hu. The fft moving average (fft-ma) generator: An efficient numerical method for generating and conditioning gaussian simulations. Mathematical Geology, 32:701-723, 2000.

[60] D. Sanchez, L. Hume, R. Chatelin, and P. Poncet. Analysis of the 3D non-linear Stokes problem coupled to transport-diffusion for shearthinning heterogeneous microscale flows, applications to digital rock physics and mucociliary clearance. ESAIM: Mathematical Modelling and Numerical Analysis, 53:1083-1124, 2019.

[61] I.F. Sbalzarini, J.H. Walther, B. Polasek, P. Chatelain, M. Bergdorf, S. E. Hieber, E. M. Kotsalis, and P. Koumoutsakos. A software framework for the portable parallelization of particle-mesh simulations. In Wolfgang E. Nagel, Wolfgang V. Walter, and Wolfgang Lehner, editors, EuroPar 2006 Parallel Processing, pages 730-739, Berlin, Heidelberg, 2006. Springer Berlin Heidelberg.

[62] B. Schrader, S. Reboux, and Sbalzarini; I. F. Discretization correction of general integral pse operators for particle methods. Journal of Computational Physics, 229(11):4159 - 4182, 2010.

[63] C. Soulaine, S. Roman, A. Kovscek, and H. Tchelepi. Mineral dissolution and wormholing from a pore-scale perspective. Journal of Fluid Mechanics, 827:457 - 483, September 2017.

[64] C. Soulaine, S. Roman, A. Kovscek, and H. Tchelepi. Pore-scale modelling of multiphase reactive flow: application to mineral dissolution with production of $\mathrm{CO}_{2}$. Journal of Fluid Mechanics, 855:616-645, 2018.

[65] Vitaliy Starchenko and Anthony J. C. Ladd. The development of wormholes in laboratory-scale fractures: Perspectives from threedimensional simulations. Water Resources Research, 54(10):7946-7959, 2018.

[66] G. Strang. On the construction and comparison of difference schemes. SIAM Journal on Numerical Analysis, 5:506-517, 1968.

[67] P. Swarztrauber and R. Sweet. Efficient FORTRAN subprograms for the solution of elliptic partial differential equations. SIGNUM Newsl., 10(4), December 1975

[68] D. Trebotich and D. T. Graves. An adaptive finite volume method for the incompressible navier-stokes equations in complex geometries. Comm. App. Math. Comp. Sci., 10(1):43-82, 2015.

[69] F. J. Valdes-Parada, J. Alberto Ochoa-Tapia, and J. Alvarez-Ramirez. On the effective viscosity for the darcy-brinkman equation. Physica A: Statistical Mechanics and its Applications, 385(1):69 - 79, 2007.

[70] N. Wakao and J. M. Smith. Diffusion in catalyst pellets. Chemical Engineering Science, 17(11):825 - 834, 1962.

[71] J. F. Wehner and R. H. Wilhelm. Boundary conditions of flow reactor. Chemical Engineering Science, 6(2):89 - 93, 1956.

[72] S. Whitaker. The Method of Volume Averaging. Springer Berlin Heidelberg, 1999.

[73] G. S. Winckelmans, A. A. Wray, O. V. Vasilyev, and H. Jeanmart. Explicit-filtering large-eddy simulation using the tensor-diffusivity model supplemented by a dynamic smagorinsky term. Physics of Fluids, 13(5):1385-1403, 2001. 